\title{
Time-crystalline behavior in an engineered spin chain
}

\author{
Robin Schäfer,, , 2, f Götz S. Uhrig, 1 † and Joachim Stolze ${ }^{1, \text { 用 }}$ \\ ${ }^{1}$ Technische Universität Dortmund, Fakultät Physik, D-44221 Dortmund, Germany \\ ${ }^{2}$ Max-Planck-Institut für Physik komplexer Systeme, \\ Nöthnitzer Str. 38, D-01187 Dresden, Germany
}

(Dated: January 18, 2023)

\begin{abstract}
Time crystals break the discrete time translational invariance of an external periodic drive by oscillating at an integer multiple of the driving period. In addition to this fundamental property, other aspects are often considered to be essential characteristics of a time crystal, such as the presence of disorder or interactions, robustness against small variations of system parameters and the free choice of the initial quantum state. We study a finite-length polarized XX spin chain engineered to display a spectrum of equidistant energy levels without drive and show that it keeps a spectrum of equidistant Floquet quasienergies when subjected to a large variety of periodic driving schemes. Arbitrary multiples of the driving period can then be reached by adjusting parameters of the drive, for arbitrary initial states. This behavior is understood by mapping the XX spin chain with $N+1$ sites to a single large spin with $S=N / 2$ invoking the closure of the group SU(2). Our simple model is neither intrinsically disordered nor is it an interacting many-body system (after suitable mapping), and it does not have a thermodynamic limit in the conventional sense. It does, however, show controllable discrete time translational symmetry breaking for arbitrary initial states and a degree of robustness against perturbations, thereby carrying some characteristic traits of a discrete time crystal.
\end{abstract}

\section{INTRODUCTION}

Over the last decades, out of equilibrium systems have drawn increasing attention. Especially, low dimensional quantum systems with an external periodic drive have been investigated under different aspects. The results obtained exhibit phenomena that are intensively discussed in the condensed matter community. In particular, the concepts of discrete time crystals or Floquet time crystals and the associated broken time translation symmetry in periodically driven systems have been studied in both theory and experiment.

The initial suggestion 1 of a time-crystalline ground state was quickly proved wrong $2 \sqrt{4}$. In an equilibrium system described by a time-independent Hamiltonian, timetranslation symmetry breaking (TTSB) cannot show up in any observable since the expectation value of any Heisenberg operator $O(t)=e^{i H t} O e^{-i H t}$ (we set $\hbar=1$ throughout) is time-independent. Consequently, TTSB can only be detected in the behavior of correlation functions. Given some local quantity $C(x, t)$ it was suggested $\sqrt[5]{ }$ that the infinite-volume limit

$$
\lim _{V \rightarrow \infty}\langle C(x, t) C(0,0)\rangle=f(x, t)
$$

should show non-trivial periodically oscillating longrange order for the system to be called a time crystal. After the existence of time crystals of this kind was ruled out ${ }^{5}$ by Watanabe and Oshikawa for equilibrium systems the idea was picked up by others ${ }^{6 / 2}$ and generalized to periodically driven systems. The external drive imposes a discrete time translation symmetry which may be broken by the system showing periodicity at a nontrivial integer multiple of the driving period. This phenomenon has come to be known as Floquet time crystal ${ }^{11 \mid 13}$ or discrete time crystat $\sqrt{9[12[14 \sqrt{16}}$ (DTC). Experimental evidence for that kind of TTSB has been reported for suitably prepared initial states.

The concept of Floquet time crystals has developed only recently and is still intensely debated; there are

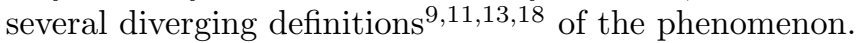
Most of these refer to a finite domain in both space and time, hence we refer to these systems as DTCs on a finite level. All definitions require the original TTSB, but additional conditions vary. A discrete time crystal on a finite level is a robust and periodically driven quantum mechanical system that exhibits TTSB for some (specific) initial states $\left|\Psi_{0}\right\rangle$ :

$$
\left|\Psi_{0}\right\rangle=e^{i \varphi} U(n T)\left|\Psi_{0}\right\rangle, \quad \text { for some integer } \quad n \geq 2,
$$

where $U(t)=U(t+T)$ is the periodic time evolution operator and $\varphi$ is a global phase. The desired robustness here refers to variations in (i) the initial state or (ii) the parameters in the Hamiltonian of the system. Definitions of DTC beyond the finite level may either include the thermodynamic limit $V \rightarrow \infty$ or demand the stability of the system for $t \rightarrow \infty$, or both.

Interestingly, the concept of discrete time-translation symmetry in driven quantum systems was studied long before the advent of time crystals. For example, Dunlap and Kenkre ${ }^{19}$ discovered dynamic localization in a periodically driven one-dimensional tight-binding system in 1986. The irregular dynamics of the system turns periodic when suitably driven, hence the stroboscopic dynamics is frozen and a localized state stays localized under stroboscopic observation.

In classical nonlinear dynamics the breaking of an externally imposed discrete time-translation symmetry is well known, manifesting itself in phenomena like parametric resonance or the occurrence of subharmonics in 
simple driven nonlinear systems 20 . DTCs may be viewed as the quantum generalizations of these phenomena.

It has frequently been argued that disorder or interaction (or both) are necessary ingredients for DTCs. However, recent studies ${ }^{13|14| 16}$ have shown that disorder is not necessary. Here we are going to present a simple system lacking both disorder and interaction which nevertheless displays DTC-like breaking of time-translation symmetry. The model system is a spin chain with engineered nearest-neighbor couplings 21 subject to a periodic drive. The coupling constants are fixed in such a way as to make the dynamics of the spin chain periodic without external drive. The drive then introduces an additional time scale and the interplay between the two time scales may lead to the breaking of the time-translation symmetry defined by the drive.

A Jordan-Wigner transformation ${ }^{22}$ maps spin excitations in the system to non-interacting fermions. We use analytical and numerical techniques to show that the system can behave periodically at any arbitrary nontrivial multiple of the driving period. Since periodicity is established on the level of the time evolution operator, the condition (2) is fulfilled for arbitrary initial states $\left|\Psi_{0}\right\rangle$ once two global parameters of the Hamiltonian are properly adjusted. In the limit of an infinitely long quantum spin chain the analysis of the quantum dynamics carries over to a driven classical system without any changes and the breaking of time-translation symmetry seamlessly connects to classical subharmonic behavior.

The structure of this paper is as follows. Sec. II discusses the formal details of TTSB in a periodically driven system, focusing on systems with a simple equidistant spectrum of Floquet quasienergies. Depending on commensurability or resonance conditions between the quasienergy level spacing and the driving frequency, time-translation symmetry can be conserved as well as broken in the manner of a DTC. Subsequently, Sec. III treats the special case of a periodically time-dependent Hamiltonian consisting of mutually commuting Fourier components. Sec. IV is the central part of this paper, containing results on a driven finite spin- $1 / 2 \mathrm{XX}$ chain with engineered nearest-neighbor couplings and a sitedependent $z$ magnetic field which varies linearly along the chain. That spin chain is equivalent to non-interacting fermions with nearest-neighbor hopping and a linearly varying local potential.

The detailed results on the dynamics of that system in the absence of driving are used to study the behavior under a binary drive, where the slope of the magnetic field (or local potential) is periodically switched between two values. We show that TTSB for arbitrary initial states can be achieved at arbitrary multiples of the driving period by adjusting the parameters of the drive. The binary drive is not the only way to achieve TTSB; a harmonic (sinusoidal) drive is one of many other possibilities. In that case, however, numerical Floquet techniques must be used to obtain results similar to those obtained for the binary drive. Numerical observations show that the time-translation symmetry-broken state is robust against local perturbations of the system parameters, at least for not too long times. This robustness consists in the preservation of the peak in the Fourier transform of dynamic correlations at the subharmonic frequency, see below, although its spectral weight is reduced gradually upon increasing disorder. Additionally, we discuss to which extent an ideal time-crystalline system can display heating. Sec. V] contains concluding remarks and points out possible applications in quantum information processing.

\section{TIME TRANSLATION SYMMETRY BREAKING}

As outlined in the preceding section, the fundamental feature of a discrete time crystal is commensurate TTSB. Here, we discuss under which conditions periodic behavior can be established in periodically driven systems, using the framework of Floquet theory 23.27 .

To start, we consider a time-independent system, described by a Hamiltonian $H$ with eigenstates $\left|\varphi_{\alpha}\right\rangle$ and eigenvalues $E_{\alpha}$. The time evolution operator is given by

$$
U(t)=\sum_{\alpha} e^{-i t E_{\alpha}}\left|\varphi_{\alpha}\right\rangle\left\langle\varphi_{\alpha}\right|
$$

The system then shows periodic behavior with period $T_{S}$, (apart frome a global phase $\varphi$ ) if for all $\alpha$

$$
T_{S} E_{\alpha}=2 \pi m_{\alpha}-\varphi
$$

with integers $m_{\alpha}$.

For a periodically driven system, $H(t+T)=H(t)$, Floquet theory 23 shows that a general solution of the Schrödinger equation is a superposition of timedependent states

$$
\left|\Psi_{\alpha}(t)\right\rangle=\rho_{\alpha}^{t / T}\left|\Phi_{\alpha}(t)\right\rangle=e^{-i \varepsilon_{\alpha} t}\left|\Phi_{\alpha}(t)\right\rangle .
$$

Here, the Floquet multipliers $\rho_{\alpha}$ are uniquely determined, while the quasienergies $\varepsilon_{\alpha}$ are only defined modulo $\omega=2 \pi / T$ and thus can be restricted by $-\omega / 2 \leq \varepsilon_{\alpha}<$ $\omega / 2$. The Floquet multipliers form the spectrum of the time evolution operator over one period, $U(T)$. Their absolute values equal unity since the time evolution is unitary. The Floquet modes $\left|\Phi_{\alpha}(t)\right\rangle=\left|\Phi_{\alpha}(t+T)\right\rangle$ are periodic with period $T$ and form a complete orthogonal set for all $t$. From (5) we may construct the evolution operator:

$$
U(t)=\sum_{\alpha} e^{-i \varepsilon_{\alpha} t}\left|\Phi_{\alpha}(t)\right\rangle\left\langle\Phi_{\alpha}(0)\right|,
$$

with obvious similarities to the time independent case (3). Periodic behavior ensues if the exponentials share a common period $T_{S}$ commensurate to the period $T$ of the drive, and hence, of the Floquet modes. That is the case if for all $\alpha$

$$
T_{S} \varepsilon_{\alpha}=2 \pi m_{\alpha}-\varphi
$$


with integers $m_{\alpha}$ and one global phase $\varphi$, and if there is a positive integer $n$ such that

$$
T_{S}=n T .
$$

Thus, only one additional condition (8) is necessary to achieve periodic behaviour in periodically driven systems as compared to the time independent case (4). The condition (7) implies that every Floquet multiplier $\rho_{\alpha}$ equals an $n$th root of $e^{i \varphi}$. This leads to $\rho_{\alpha}^{T_{S} / T}=\rho_{\alpha}^{n}=e^{i \varphi}$ and, by (6), to periodicity with period $T_{S}=n T$.

Given a driving frequency $\omega$ and a finite set of quasienergies $\varepsilon_{\alpha}$ it is always possible to approximately fulfill conditions $(7 \overline{8})$ for some time $T_{S}$ to some degree of accuracy. Unfortunately, however, $T_{S}$ will grow exponentially with the number of conditions, or quasienergies. Symmetries in the structure of the quasienergies would help to drastically reduce the number of independent conditions $(7 / 8)$ and to find serious candidates for Floquet time crystals.

One suitable scenario consists in having an integer spectrum of quasienergies, meaning that all quasienergies $\varepsilon_{\alpha}$ are integer multiples of some characteristic value $\varepsilon_{0}: \varepsilon_{\alpha}=\varepsilon_{0} \alpha$ with $\alpha \in \mathbb{Z}$. In this case, the many conditions $(78)$ collapse to just two with $\varphi=0$, namely

$$
\begin{aligned}
T_{S} \varepsilon_{0} & =2 \pi m_{0} \\
T_{S} & =n T
\end{aligned}
$$

for integer $m_{0}$ and $n$. The two conditions (9) ensure periodicity even if some quasienergies $\varepsilon_{\alpha}$ lie outside the first "Brillouin zone" in time $[-\omega / 2, \omega / 2)$ and thus must be shifted by a multiple of $\omega$; in that case $9 \mathrm{~b}$ makes sure that (7) holds.

It should be noted that an integer spectrum alone, with some arbitrary value of $\varepsilon_{0}$, does not ensure periodicity for any reasonable time $T_{S}$, as illustrated in figure 1 . There, neither the quasienergies (reduced to the first Brillouin zone) nor the Floquet multipliers display the necessary regular structures.

Periodic behavior ensues, however, if $\varepsilon_{0}$ is chosen such as to satisfy certain commensurability or resonance conditions; two cases can be distinguished here. In case I the spectrum of quasienergies collapses, since

$$
\varepsilon_{0}=0 .
$$

In that case all Floquet multipliers $\rho_{\alpha}=1$ and the system displays the periodicity of the Floquet modes $\left|\Phi_{\alpha}(t)\right\rangle$, hence $T_{S}=T$ and time-translation symmetry is conserved. In periodically driven lattice systems whose undriven dynamics displays Bloch oscillations this situation has been discussed under the label of Bloch band collapse $^{28}$; dynamic localization ${ }^{19 / 29 \mid 30}$ also involves a band collapse. The key feature is the synchronisation between the drive and the Bloch oscillations such that within every period of the drive the system performs an integer number of Bloch oscillations.

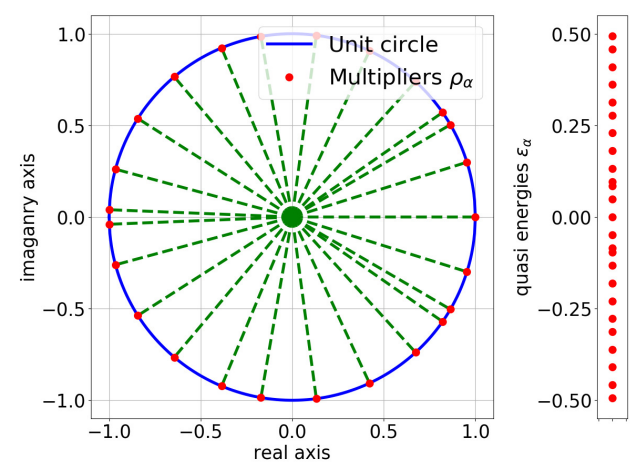

FIG. 1: Floquet multipliers (left) and quasienergies (right) for an integer spectrum $\varepsilon_{\alpha}=\varepsilon_{0} \alpha \bmod \omega$ of 25 quasienergies. $\left(\alpha=-24,-22, \ldots, 24, \varepsilon_{0}=0.13, \omega=1\right.$.) The incommensurate structure of the Floquet multipliers prevents periodic behavior at any reasonable multiple of the driving period.

More interesting behavior is displayed by the resonance case II, where

$$
\left|\varepsilon_{0}\right|=m / n \omega / 2
$$

and $1 \leq m<n$ and $m$ is not a divisor of $n$. This case leads to periodic behavior with period $T_{S}=n T$, provided the set of integers $\alpha$ defining the spectrum of quasienergies $\varepsilon_{\alpha}=\alpha \varepsilon_{0}$ contains either only even or only odd numbers; otherwise the time evolution leads to interference between terms with phases zero and $\pi$, respectively, at $t=T_{S}$. If the quasienergy spectrum contains both even and odd integers $\alpha$, constructive interference occurs at multiples of $2 T_{S}$. Case II is of interest since it breaks time-translation symmetry at period $T_{S}=n T$. We illustrate this case for $n=3$ in figure 2, All Floquet multipliers are third roots of unity and the reduced quasienergies assume only three different values. This scenario was already observed in a driven interacting one-dimensional system $^{30}$, where dynamic localization leads to an integer spectrum, allowing for both periodic and quasiperiodic behavior.

\section{COMMUTING FOURIER HAMILTONIANS}

Under mild conditions, a $T$-periodic Hamiltonian may be written as a Fourier series

$$
H(t)=\sum_{n \in \mathbb{Z}} e^{-i n \omega t} H_{n}
$$

The situation is especially simple if all Fourier coefficients commute

$$
\left[H_{n}, H_{m}\right]=0 .
$$

The Hamiltonian then commutes with itself for different times

$$
\left[H(t), H\left(t^{\prime}\right)\right]=0 .
$$




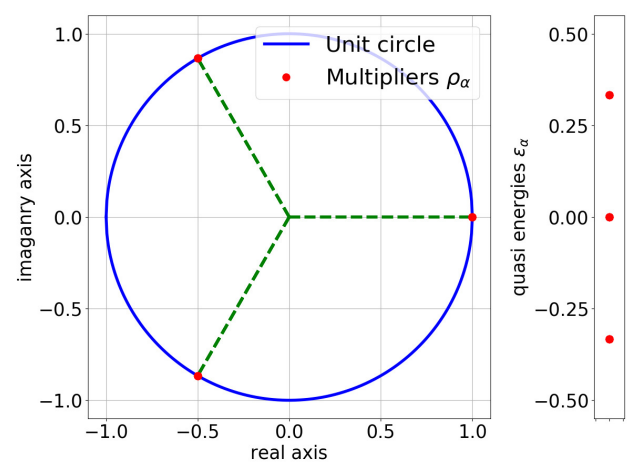

FIG. 2: Same as Fig. 1, for $\alpha=-24,-22, \ldots, 24$, $\varepsilon_{0}=1 / 6, \omega=1$. Both Floquet multipliers and reduced quasienergies show commensurate structures leading to a time-translation symmetry breaking period $T_{S}=3 T$.

It is then possible to induce TTSB if $H$ contains a stationary part and impossible if not, as we will show presently. Subsequently we will discuss a simple harmonic drive as an important special case.

The spectrum of the time evolution operator over one period $T$ determines the Floquet multipliers and hence, the quasienergies. Due to commutativity (14) the evolution operator is simply

$$
\begin{aligned}
U(T) & =\exp \left(-i \int_{0}^{T} \sum_{n \in \mathbb{Z}} e^{-i n \omega \tau} H_{n} \mathrm{~d} \tau\right) \\
& =\exp \left(-i T H_{0}\right) .
\end{aligned}
$$

Its Floquet multipliers are given by $e^{-i T E_{\alpha}^{0}}$ where $E_{\alpha}^{0}$ are the eigenenergies of the stationary Hamiltonian $H_{0}$. Hence, the quasienergies are given by $E_{\alpha}^{0}$, backfolded into $[-\omega / 2, \omega / 2)$. If the eigenenergies of $H_{0}$ form an integer spectrum and fulfill the resonance condition (11) the system breaks time-translation symmetry with pe$\operatorname{riod} T_{S}=n T$, as discussed in the previous section.

If, on the other hand, $H(t)$ does not contain a stationary part, that is, $H_{0}=0$, the quasienergy spectrum collapses to zero and time-translation symmetry is conserved.

A stationary Hamiltonian driven by a purely sinusoidal perturbation is a commonly encountered situation in physics

$$
H(t)=H_{0}+2 \cos (\omega t) V .
$$

If $\left[H_{0}, V\right]=0,16$ is one of the simplest commuting Fourier Hamiltonians.

The example offers an opportunity to illustrate how the Floquet formalism works in a transparent situation. We refer to some basic notions of the Floquet formalism; more details can be found in the literature ${ }^{24 \mid 31}$. Since the Floquet modes $\left|\Phi_{\alpha}(t)\right\rangle$ are $T$-periodic they can be expanded in a Fourier series of Hilbert space vectors, which in turn can be expanded in a suitable basis. Choosing the eigenvectors of $H_{0}$ as that basis and assuming (for the ease of discussion) that $H_{0}$ acts on a $D$-dimensional Hilbert space, we obviously have to determine a $D$-dimensional vector for each Fourier component. The $T$-periodic drive, when expanded in a Fourier series itself, connects different Fourier components of the Floquet modes. Thus, the Floquet modes are eigenvectors of an infinite matrix consisting of $D \times D$ blocks, the Floquet matrix. The diagonal blocks of the Floquet matrix are given by $H_{0}+m \omega \mathbf{1}$ (where $\mathbf{1}$ denotes the $D \times D$ unit matrix, and $-\infty<m<\infty$ refers to the Fourier modes), while the off-diagonal blocks contain the Fourier components of the drive $V$. The eigenvalues of the Floquet matrix are the quasienergies.

For the situation considered here, the Floquet matrix is block-tridiagonal, where $H_{0}$ determines the diagonal blocks, and $V$ is contained in the off-diagonal blocks. Since $\left[H_{0}, V\right]=0$, mutual eigenstates are available:

$$
H_{0}|\alpha\rangle=E_{\alpha}^{H_{0}}|\alpha\rangle \text { and } V|\alpha\rangle=E_{\alpha}^{V}|\alpha\rangle .
$$

As mentioned before, the Floquet multipliers are determined by the eigenvalues of $H_{0}, \rho_{\alpha}=e^{-i E_{\alpha}^{H_{0}} T}$. The tridiagonal structure of the Floquet matrix maps to a three-term recursion relation fulfilled by Bessel functions. Hence, the linearly independent solutions (5) in this case are

$$
\begin{aligned}
\rho_{\alpha}^{t / T}\left|\Phi_{\alpha}(t)\right\rangle & =e^{-i E_{\alpha}^{H_{0}} t} \sum_{n \in \mathbb{Z}} e^{i n \omega t} J_{n}\left(x_{\alpha}\right)|\alpha\rangle \\
& =e^{-i\left(E_{\alpha}^{H_{0}} t-x_{\alpha} \sin \omega t\right)}|\alpha\rangle .
\end{aligned}
$$

Here, the $J_{n}$ are Bessel functions of the first kind, $x_{\alpha}:=$ $2 E_{\alpha}^{V} / \omega$, and $|\alpha\rangle$ is an eigenstate from (17). The timedependent states (18a) are mutually orthogonal and normalized whenever the $|\alpha\rangle$ are. The result $(18 \mathrm{~b})$ can actually be obtained more easily by a direct solution of the Schrödinger equation thanks to (17). However, this example clearly shows the power of the Floquet approach to periodically driven systems.

\section{DRIVEN SPIN CHAIN}

In most physically relevant driven systems the Hamiltonians at different times do not commute with each other. Hence the Floquet multipliers (5) of a periodically driven system will depend on the drive, in contrast to the situation of Sec. III. We will focus on systems which are engineered to generate a simple structure of the energy and quasienergy spectra such that time-translation symmetry breaking becomes possible. We will first discuss a binary drive, that is, the Hamiltonian $H(t)$ will be piecewise constant, alternating between two values. Later that binary drive will be replaced by a sinusoidal one, that is, the system will be harmonically driven. In both cases we will observe time-translation symmetry breaking with a period $T_{S}=n T$ which can be adjusted by changing the parameters of the system. Before driving the system, however, we discuss its properties without drive. 


\section{A. Dynamics of the undriven system}

We are considering a spin- $1 / 2 \mathrm{XX}$ chain ${ }^{32[33}$ of $N+1$ sites $i=0, \ldots, N$. A Jordan-Wigner transformation ${ }^{22}$ maps the system to noninteracting spinless lattice fermions, such that a spin-up state corresponds to a fermion, while a spin-down state is equivalent to an empty site. The total number of up spins - or fermions is conserved, and we will exclusively consider the singleparticle sector, which is spanned by the states

$$
|i\rangle:=c_{i}^{\dagger}|0 \ldots 0\rangle=|0 \ldots 0 \underbrace{1}_{\text {site } i} 0 \ldots 0\rangle
$$

containing a single fermion, or spin-up excitation, at site $i=0, \ldots, N$. $\left(c_{i}^{\dagger}\right.$ and $c_{i}$ are local Fermi creation and annihilation operators, respectively.) The Hamiltonian in the general (driven) case contains a time-dependent nearest-neighbor hopping and a local potential

$$
H(t):=\lambda \sum_{i=0}^{N-1} J_{i}(t)\left(c_{i}^{\dagger} c_{i+1}+c_{i+1}^{\dagger} c_{i}\right)+h \sum_{i=0}^{N} h_{i}(t) c_{i}^{\dagger} c_{i} .
$$

Presently we will assume $J_{i}(t)$ and $h_{i}(t)$ to be constant in time, while in the driven case they may become periodic with period $T=2 \pi / \omega$. The parameters $\lambda$ and $h$ serve to adjust the overall energy scales of the spectrum while the $J_{i}$ and $h_{i}$ fix the detailed structure. The Hamiltonian (20) generalizes a model originally introduced 21 to achieve perfect state transfer, that is, for some time $\tau$ a state at a "sender" site $s$ is transferred to a "receiver" site $r: e^{-i H \tau}|s\rangle=|r\rangle$. Given additional symmetry properties, perfect state transfer implies periodic behavior.

We will consider the configuration defined by

$$
\begin{aligned}
J_{i} & :=\sqrt{(i+1)(N-i)}, \\
h_{i} & :=N-2 i .
\end{aligned}
$$

without the local fields $h_{i}$ the system is spatially symmetric, allowing for perfect state transfer ${ }^{21}$ between the ends of the chain. The nonzero fields $h_{i}(21 \mathrm{~b})$ break the spatial symmetry. This system has been studied under various aspects 3437 .

The Hamiltonian 20 with time independent parameters (21) can be diagonalized analytically 37 . The eigenvectors are related to the orthogonal and normalized (discrete) Krawtchouk polynomials ${ }^{38} \kappa_{n}^{p}(x)$ for $0<p<1$ defined in appendix $\mathrm{A}$. The parameter $p$ depends on the scaling factors for the nearest-neighbor hopping and the field in 20 .

$$
p_{ \pm}=\frac{1}{2}\left(1 \pm \sqrt{\frac{h^{2}}{h^{2}+\lambda^{2}}}\right) .
$$

Here, $p_{+}$corresponds to $h<0$, that is, the local fields along the chain increase from $i=0$ to $N$; vice versa, $p_{-}$is appropriate if $h>0$. The crucial property of the system causing periodic dynamics is the equidistant spectrum of eigenenergies $E_{x}(x=0, \ldots, N)$

$$
E_{x}=\mu_{0}(N-2 x), \text { with } \mu_{0}:=\sqrt{\lambda^{2}+h^{2}} .
$$

The set of integers defining the spectrum will be denoted

$$
\mathcal{A}:=\{-N,-(N-2), \ldots, N-2, N\}
$$

for further reference. Note that the set of local field coefficients $h_{i}(21 \mathrm{~b}$ coincides with $\mathcal{A}$. The set $\mathcal{A}$ contains either only even or only odd integers, depending on $N$. The spectrum (23) implies that the dynamics of the system is periodic (apart from a global phase) with period

$$
T_{\mathrm{nd}}:=\frac{\pi}{\mu_{0}}=\frac{\pi}{\sqrt{\lambda^{2}+h^{2}}},
$$

where the index "nd" is short for "no drive". The eigenvectors of the Hamiltonian are given by

$$
\left|\varphi_{x}\right\rangle=\left(\kappa_{0}^{p}(x), \kappa_{1}^{p}(x), \ldots, \kappa_{N}^{p}(x)\right)^{T}
$$

for $x=0, \ldots, N$ due to the three-term recurrence relation A5b in $n$ fulfilled by the Krawtchouk polynomials $\kappa_{n}^{p}(x)$. Further properties of the $\kappa_{n}^{p}(x)$ can be used to construct the transmission amplitudes for an excitation travelling from site $s$ (sender) to site $r$ (receiver):

$$
\begin{aligned}
f_{r s}^{p}(t)= & \left\langle r\left|e^{-i H t}\right| s\right\rangle=\left\langle r\left|\sum_{x=0}^{N} e^{-i t E_{x}}\right| \varphi_{x}\right\rangle\left\langle\varphi_{x}|| s\right\rangle \\
= & e^{-i t \mu_{0} N} \sqrt{\left(\begin{array}{c}
N \\
r
\end{array}\right)\left(\begin{array}{c}
N \\
s
\end{array}\right)}[\sqrt{p(1-p)}]^{r+s} \\
& \times[1-\Gamma]^{r+s}[1-p+p \Gamma]^{N-r-s} \\
& \times{ }_{2} F_{1}\left[\begin{array}{c}
(-r,-s) \\
(-N)
\end{array} ;-\frac{\Gamma}{p(1-p)(1-\Gamma)^{2}}\right],
\end{aligned}
$$

where $\Gamma:=e^{i t 2 \mu_{0}}$ and ${ }_{2} F_{1}$ is the classical hypergeometric series 39 . Summation formulas for hypergeometric functions $s^{39]}$ can be used to derive ${ }^{36 / 37}$ the result (27), as explained in appendix A. Note that the somewhat awkward-looking formula (27) contains the complete information on the dynamics for arbitrary times and arbitrary initial states.

There is, however, a different and physically more appealing way to derive the transmission amplitudes (27). It was noted early on 2135 that the $N+1$ states $|i\rangle|19\rangle$ may be identified with the $S_{z}$ eigenstates $|m\rangle$ of a spin$N / 2$ system

$$
S_{z}|m\rangle=m|m\rangle \text { with } m=i-\frac{N}{2} .
$$

Using the relations

$$
S_{ \pm}=S_{x} \pm \mathrm{i} S_{y}
$$


and

$$
S_{ \pm}|m\rangle=\sqrt{S(S+1)-m(m \pm 1)}|m \pm 1\rangle
$$

for a spin- $S$ system, the Hamiltonian with parameters (21) maps to

$$
H=2 \lambda S_{x}-2 h S_{z}
$$

since the hopping amplitudes $J_{i}$ correspond to the matrix elements of $S_{x}$ between eigenstates $|m\rangle$ of $S_{z}$. Clearly then the dynamics induced solely by the $J_{i}$ is equivalent to that of a spin- $N / 2$ system in a magnetic field along the $x$ axis. If this large spin is initially prepared in a state with $S_{z}=-S=-N / 2$ it precesses around the $x$ axis to $S_{z}=+S$ and then back to the initial state, provided the field $h=0$ in $(20)$ or (31). This situation corresponds to the perfect transfer of a single-particle excitation from one end of the $(N+1)$-site chain to the other end and back again. For $h \neq 0$ in 20 the $h_{i}(21 \mathrm{~b})$ define a magnetic field in $z$ direction in the large spin picture (31). The large spin then precesses around an axis tilted away from the $x$ direction. Consequently the initial state $S_{z}=-S$ can never reach its antipode $S_{z}=+S$ and hence there is no more perfect state transfer, but still periodic behavior. Within the large spin analogy the transmission amplitudes 27 can be re-derived ${ }^{37}$ using the properties of the group $S U(2)$ represented by the spin operators. Note that the coincidence between the set $\mathcal{A}(24)$ and the set of coefficients $h_{i}(21 b)$ is natural in the large spin picture, since all spin operators possess equidistant spectra of eigenvalues.

To summarize this subsection, the amplitudes 27$]$ define the matrix representation of the time evolution operator of the undriven system and thus determine the dynamics completely. For $h=0$, perfect state transfer occurs at time $T_{\text {nd }} / 2$. With broken spatial symmetry, $h \neq 0$, perfect state transfer is not possible any longer while periodic behavior persists. Similar timeindependent systems, with integer spectra and consequently, periodic behavior, have been studied $34 / 40-47$ in various contexts during recent years.

\section{B. Binary driving}

We now subject the spin chain to a periodic drive via the local fields, that is,

$$
H(t):=\lambda \sum_{i=0}^{N-1} J_{i}\left(c_{i}^{\dagger} c_{i+1}+c_{i} c_{i+1}^{\dagger}\right)+f(t) h \sum_{i=0}^{N} h_{i} c_{i}^{\dagger} c_{i},
$$

with $f(t)=f(t+T)$. Specifically we apply a binary drive

$$
f(t)=\left\{\begin{array}{ll}
+1, & \text { for } 0 \leq t<T / 2 \\
-1, & \text { for } T / 2 \leq t<T
\end{array} .\right.
$$

Note that the time average $\overline{f(t)}=0$ is such that the spatial symmetry of the system is restored on average.
As with other piecewise time-independent models ${ }^{30}$ the time evolution of the binarily driven system is most easily discussed without using the Floquet matrix formalism. Since the Hamiltonian is piecewise constant in time, the time evolution operator can be assembled as a product, using the results of Sec. IV A During the interval $[0, T / 2)$ the local fields decrease from site $i=0$ to $N$, hence the transmission amplitudes (27) depend on the parameter $p_{-}$from (22). During the second half $[T / 2, T)$ of the period, $p_{+}$is the relevant parameter in (27). Denoting by $U_{ \pm}(T / 2)$ the time evolution operators over the two half-periods, the evolution operator over the full period $[0, T)$ can be written as

$$
U(T)=U_{+}(T / 2) U_{-}(T / 2) .
$$

Since the matrix elements

$$
\left\langle r\left|U_{ \pm}(T / 2)\right| s\right\rangle=f_{r s}^{p_{ \pm}}(T / 2)
$$

are known from (27) we can use product formulas for hypergeometric functions to determine the elements of $U(T)$

$$
\begin{aligned}
u_{r s}(T)= & \langle r|U(T)| s\rangle=\sum_{k=0}^{N} f_{r k}^{p_{+}}(T / 2) f_{k s}^{p_{-}}(T / 2) \\
= & e^{-i \mu_{0} N T} \sqrt{\left(\begin{array}{c}
N \\
r
\end{array}\right)\left(\begin{array}{c}
N \\
s
\end{array}\right)}\left[\frac{2 \sqrt{p_{+} p_{-}}(1-\Gamma)}{\Gamma+2 p_{+} p_{-}(1-\Gamma)^{2}}\right]^{r+s} \\
& \times\left[1-p_{-}+p_{-} \Gamma\right]^{r}\left[1-p_{+}+p_{+} \Gamma\right]^{s} \\
& \times\left[\Gamma+2 p_{+} p_{-}(1-\Gamma)^{2}\right]^{N}{ }_{2} F_{1}\left[\begin{array}{c}
(-r,-s) \\
(-N)
\end{array} ; \eta\right] \\
& \text { with } \Gamma:=\exp \left(i T \mu_{0}\right) \\
& \text { and } \eta:=-\frac{1}{4} \frac{\Gamma^{2}}{\Gamma+p_{+} p_{-}(1-\Gamma)^{2}} \frac{1}{p_{+} p_{-}(1-\Gamma)^{2}}
\end{aligned}
$$

see appendix $\mathrm{B}$ for details. The Floquet multipliers $\rho_{\alpha}$ (5) are the eigenvalues of $U(T)$. Unfortunately, we could not derive an analytic expression for $\rho_{\alpha}$ from (36). $\mathrm{Nu}-$ merical diagonalization of the matrix defined by (36), however, revealed integer spectra of quasienergies in all cases considered, and for all system sizes $N$ studied. This holds true even if the two operators $U_{+}$are applied for different lengths of time, that is, if (34) is replaced by $U(T)=U_{+}(\beta T) U_{-}((1-\beta) T), \quad 0<\beta<1$. In all cases, the quasienergies can be written in the form

$$
\varepsilon_{\alpha}=\varepsilon_{0} \alpha \text { for } \alpha \in \mathcal{A},
$$

where $\mathcal{A}$ is the set (24) of integers (either all even or all odd), and, importantly, the characteristic scale $\varepsilon_{0}$ does not depend on the system size $N$; this will be explained below. The quasienergy scale only depends on the driving frequency $\omega$ and the scales $h$ of the local fields, and $\lambda$ of the nearest-neighbor couplings, respectively, which determine the Hamiltonian. By means of these parameters 
$\varepsilon_{0}$ can be tuned to fulfill the resonance condition II, eq. (11). Consequently, time-translation symmetry is broken and the system behaves periodically with the adjustable period $n T$.

The observed structure of the numerical results is analytically explained within the large spin analogy defined by the Hamiltonian (31). The single-period time evolution $U(T)$ is a $S U(2)$ operation, even if the parameters $\lambda$ and $h$ in (31) are time-dependent, due to the closure of the group $S U(2)$. Hence, this holds true for the simple time-dependence given by (33) as well as for arbitrarily complex ways of driving the system periodically. For any given driving protocol the eigenvalues of $U(T)$ are given by $e^{-i T \varepsilon_{\alpha}}$ with quasienergies $\varepsilon_{\alpha}$ from (37), while the number of quasienergies is determined by the dimension of the representation of $S U(2)$, in other words, by the spin quantum number $S=N / 2$ in (31) or the chain length $N+1$. Consequently we can use the simplest twodimensional representation of $S U(2)$ to determine the "quasienergy quantum" $\varepsilon_{0}$. The driven $\mathrm{XX}$ chain then has only has two sites and $U(T)$ is a $2 \times 2$ matrix with eigenvalues $e^{ \pm i T \varepsilon_{0}}$ and trace $2 \cos \left(T \varepsilon_{0}\right)$. The two factors $U_{ \pm}$in (34) are easily calculated and from the trace of $U(T)$ we obtain

$$
\varepsilon_{0}=\frac{1}{T} \arccos \left(\frac{h^{2}+\lambda^{2} \cos \left(\mu_{0} T\right)}{h^{2}+\lambda^{2}}\right) .
$$

The group-theoretical treatment of the driven quantum-mechanical system employing the large-spin picture provides an interesting connection to classical dynamics. The equations of motion

$$
i \frac{d}{d t}\left\langle S_{\alpha}(t)\right\rangle=\left\langle\left[S_{\alpha}(t), H\right]\right\rangle, \alpha=x, y, z
$$

for the spin expectation values with $H$ given by (31) lead to the differential equation

$\frac{d^{2}}{d t^{2}}\left(\begin{array}{c}\left\langle S_{x}(t)\right\rangle \\ \left\langle S_{y}(t)\right\rangle \\ \left\langle S_{z}(t)\right\rangle\end{array}\right)=-4\left(\begin{array}{ccc}h^{2} & 0 & \lambda h \\ 0 & h^{2}+\lambda^{2} & 0 \\ \lambda h & 0 & \lambda^{2}\end{array}\right)\left(\begin{array}{c}\left\langle S_{x}(t)\right\rangle \\ \left\langle S_{y}(t)\right\rangle \\ \left\langle S_{z}(t)\right\rangle\end{array}\right)$

describing precession of the spin expectation value with angular velocity

$$
2 \sqrt{h^{2}+\lambda^{2}}=2 \mu_{0}
$$

about the axis

$$
\vec{e}=\frac{1}{\sqrt{h^{2}+\lambda^{2}}}\left(\begin{array}{c}
-\lambda \\
0 \\
h
\end{array}\right)
$$

Solving the differential equation 40 the time evolution of the "classical" spin vector $\langle\vec{S}(t)\rangle$ assumes the form

$$
\langle\vec{S}(t)\rangle=M(t, h, \lambda)\langle\vec{S}(0)\rangle
$$

with a $3 \times 3$ rotation matrix $M(t, h, \lambda)$. The time evolution under the binary driving protocol is then described by

$$
\langle\vec{S}(t)\rangle=M\left(\frac{T}{2},-h, \lambda\right) M\left(\frac{T}{2}, h, \lambda\right)\langle\vec{S}(0)\rangle .
$$

The product of the two $M$ matrices is again a rotation matrix and the rotation angle $\varphi$ can be determined from

$$
\operatorname{Tr}\left(M\left(\frac{T}{2},-h, \lambda\right) M\left(\frac{T}{2}, h, \lambda\right)\right)=1+2 \cos \varphi
$$

which leads to

$$
\cos \left(\frac{\varphi}{2}\right)=\frac{h^{2}+\lambda^{2} \cos \left(\mu_{0} T\right)}{h^{2}+\lambda^{2}} .
$$

On the one hand, time-translation symmetry in this classical picture is broken resulting in a period $n T$ if

$$
\langle\vec{S}(n T)\rangle=\langle\vec{S}(0)\rangle
$$

or

$$
n \varphi=2 \pi m
$$

for some integer $m$. On the other hand, the condition for broken time-translation symmetry in the quantum picture is

$$
U(n T)= \pm \mathbf{1}
$$

or

$$
n \varepsilon_{0} T=m \pi,
$$

since the $\operatorname{sign} e^{i m \pi}$ of the quantum state at time $n T$ is irrelevant. In view of $(38)$ and $(46)$ these conditions are equivalent.

We thus see that the time-translation symmetry breaking dynamics of the driven quantum system $\sqrt{32}$ is in a sense equivalent to the subharmonic behavior of a classical top which is parametrically driven by periodically switching its axis of precession.

By choosing the appropriate value of $\varepsilon_{0}$, the Floquet multipliers, the eigenvalues of the time evolution operator $U(T)$ over one period, can be adjusted to coincide with $n$th roots of unity and thus induce a period of $T_{S}=n T$ in the dynamics of the original quantum system. Figure 3 shows the absolute value of $\varepsilon_{0}$ (38) as a function of $h$ and $\lambda$, for $\omega=1$. Note that $\omega=1$ merely fixes the units in which energies, $\varepsilon_{0}, \lambda$, and $h$ are measured, and thus changing the driving frequency $\omega$ would merely result in a rescaling of all axes in Fig. 3

The shape shown in Fig. 3 can be understood from some simple observations. As $\lambda \rightarrow 0$ the two-site system degenerates to a pair of isolated spins and no dynamics is possible, hence $\varepsilon_{0} \rightarrow 0$. At $h=0$, there is no more drive and we are back to the stationary system from Sec. IV A with two (quasi)energy eigenvalues $\pm \lambda$. Growing $\lambda$ 


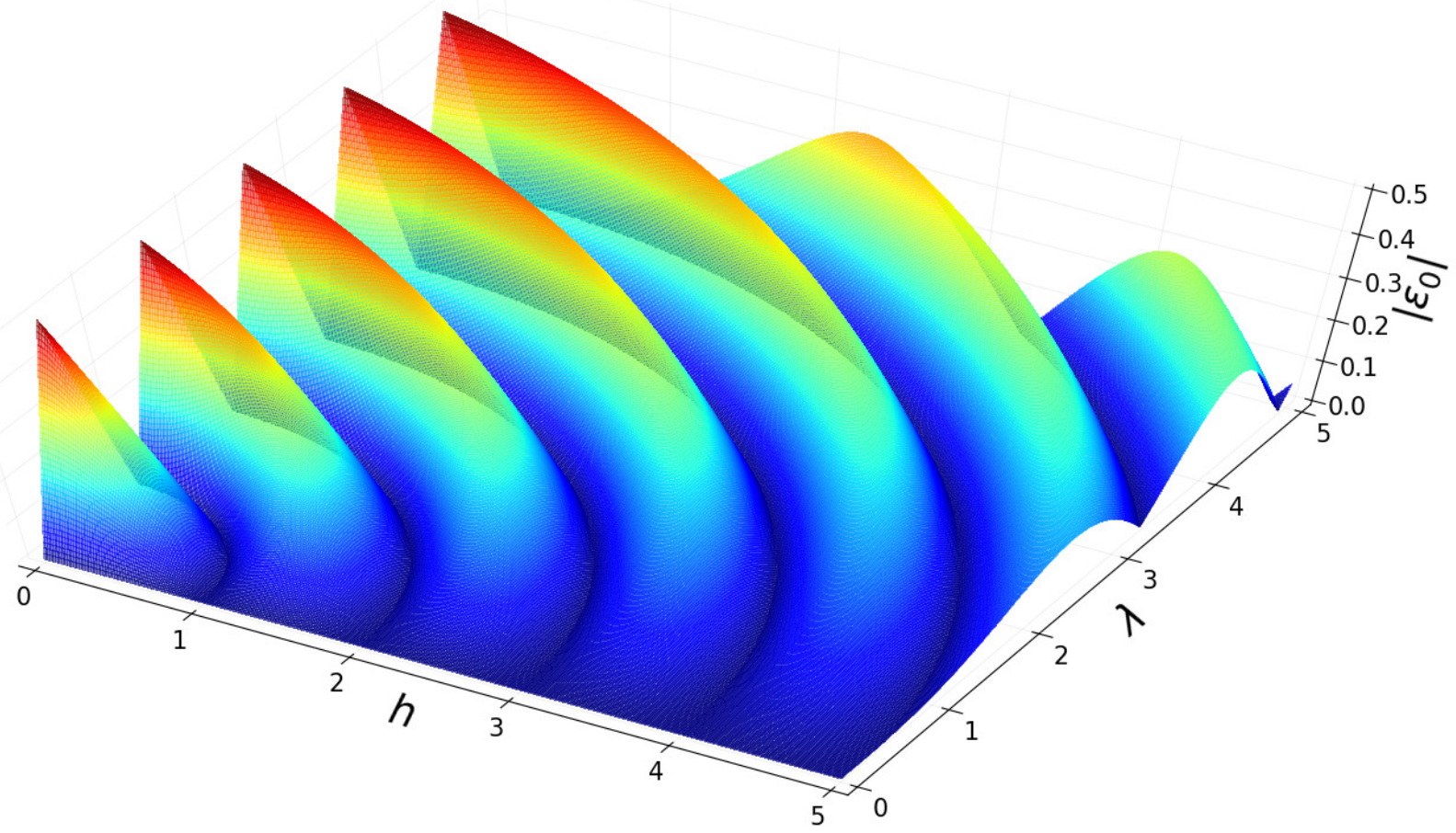

FIG. 3: Absolute value $\left|\varepsilon_{0}\right|<\omega / 2$ of the characteristic quasienergy scale (38), reduced to the first Brillouin zone in time, for binary driving with driving frequency $\omega=1$. The parameter $\lambda$ denotes the strength of the nearest-neighbor couplings and $h$ is the amplitude of time-dependent external field in the Hamiltonian (20) with coefficients 21].

then, together with the backfolding to $\left|\varepsilon_{0}\right|<\omega / 2$, leads to the regular zig-zag shape along the edge $h=0$. The tubular shapes in Fig. 3 are separated by circles in the $(\lambda, h)$ plane along which $\varepsilon_{0}=0$. These circles are defined by

$$
\mu_{0}=\sqrt{\lambda^{2}+h^{2}}=m_{0} \omega,
$$

with integer $m_{0}$, from which $\cos \left(\mu_{0} T\right)=1$ and hence $\varepsilon_{0}=0$ in (38). This is the resonance condition I (10) which implies $\rho_{ \pm}=1$, leading to periodic behavior with $T_{S}=T$; thus time-translation symmetry is conserved. The physical interpretation of this resonance is straightforward. Note that during each half-period the binarily driven system behaves as discussed in Sec. IVA, showing periodic behavior at period $T_{\text {nd }}=\pi / \mu_{0}(25)$. The condition (51) then translates to $m_{0} T_{\mathrm{nd}}=T / 2$ and hence the states of the system at the beginning and at the end of each half-period are identical. As an example, a singlespin excitation launched at site 0 will be exactly restored after each half-period. This is equivalent to the situation encountered in dynamical localization ${ }^{29130}$, where during each half-period of the drive the system performs an integer number of Bloch oscillations and consequently the single-period propagator equals unity.

The quasienergy degeneracy, $\varepsilon_{0}=0$, along circles (51) in the $(\lambda, h)$ plane was in fact already observed earlier in a two-level system subject to the binary drive (33). Numerical observation ${ }^{48}$ was followed by analytical derivation $\stackrel{49}{ }$, along with a graphical interpretation in terms of trajectories on the Bloch sphere generated by the equation of motion (39) and leading to the same conclusions as drawn in the previous paragraph.

It is also interesting to note that the binary drive (33) implies a generalized parity symmetry ${ }^{48}$. Quasienergies of Floquet modes which are even and odd under that symmetry are allowed to coincide, which is forbidden by the von Neumann-Wigner theorem $\frac{\sqrt{50}}{}$ in the absence of that symmetry. In fact, replacing the two half-periods $\frac{T}{2}$ in $(33)$ by unequal times $t_{ \pm}=\frac{T}{2}(1 \pm \alpha)$ ), the result (38) for $\varepsilon_{0}$ may be generalized, resulting in a nonzero $\varepsilon_{0}$ unless the times $t_{ \pm}$are commensurate, that is, $\alpha=\frac{p}{q}$ with integer $p$ and $q$. In that case $\varepsilon_{0}=0$ on circles of radius $q \omega, 2 q \omega$, etc.

It should be noted that the information contained in Fig. 3 is redundant, due to the periodicity of the undriven system, with period $T_{\text {nd }}(25)$. If that period is shorter than $T / 2$, the time interval during which the external drive stays constant, the system completes more than one period and some states are visited more than once. Hence it suffices to study systems with $T_{\mathrm{nd}} \geq T / 2$, which translates to

$$
\lambda^{2}+h^{2} \leq \omega^{2} .
$$

For all other parameter combinations the system merely undergoes a number of extra oscillations within each halfperiod of the drive. The simplest case of this situation, 
when (51) holds and $\varepsilon_{0}=0$, was discussed above. Interestingly, nontrivial values of $\varepsilon_{0}$ can be determined analytically, if

$$
\begin{aligned}
\left(2 m_{0}+1\right) \frac{T_{\mathrm{nd}}}{2} & =\frac{T}{2} \\
\Leftrightarrow \mu_{0}=\sqrt{\lambda^{2}+h^{2}} & =\left(m_{0}+\frac{1}{2}\right) \omega,
\end{aligned}
$$

that is, on the ridges between the valleys (51) in Fig. 3. Condition (53a) means that for this combination of $\lambda$ and $h$, a single-spin excitation started at site 0 reaches the turning point of its periodic motion precisely after a half-period of the drive. From (53b) $\cos \left(\mu_{0} T\right)=-1$ and since $\lambda$ and $h$ can be expressed in polar coordinates

$$
\left(\begin{array}{l}
\lambda \\
h
\end{array}\right)=\mu_{0}\left(\begin{array}{l}
\cos (\varphi) \\
\sin (\varphi)
\end{array}\right), \text { for } \varphi \in(0, \pi / 2),
$$

(38) simplifies to

$$
\begin{aligned}
T \varepsilon_{0} & =\arccos \left(\frac{h^{2}-\lambda^{2}}{h^{2}+\lambda^{2}}\right) \\
& =\arccos \left(\sin (\varphi)^{2}-\cos (\varphi)^{2}\right)=\pi-2 \varphi
\end{aligned}
$$

Since $\varphi$ varies between 0 and $\pi / 2, \varepsilon_{0}$ can take any value between 0 and $\omega / 2$, consequently, the resonance condition (11) can be fulfilled for arbitrary $n$ and then timetranslation symmetry is commensurately broken with the period $n T$. Given a desired period $n T$ we can use the following simple explicit prescription to break timetranslation symmetry with that period: (i) pick $\mu_{0}$ according to 53b with some suitable integer $m_{0}$, (ii) pick

$$
\varphi=\frac{\pi}{2}\left(1-\frac{m}{n}\right),
$$

where $m$ is some integer less than $n$ and not a divisor of $n$, and finally (iii) adjust $\lambda$ and $h$ according to (54).

The results described above were based on the integer structure (37) of the quasienergy spectrum which holds for all system sizes due to the $S U(2)$ symmetry of the driven $N+1$-site chain which is equivalent to a spin $N / 2$ driven by a time-dependent field.

We now focus on some results which can be obtained from the explicit matrix form (36) of the propagator $U(T)$. In order to detect $T$-periodic behavior we study the return probability $\left|u_{00}(T)\right|$ of a localized excitation initially prepared at site 0 using the transmission amplitudes $u_{r s}(T)$ (36). Perfect periodic return, $\left|u_{00}(T)\right|=1$, is obtained if and only if

$$
\begin{aligned}
& \left|\left[\Gamma+2 p_{+} p_{-}(1-\Gamma)^{2}\right]\right| \\
= & \left|\left[1-\frac{\lambda^{2}}{\lambda^{2}+h^{2}}\left(1-\cos \left(\mu_{0} T\right)\right)\right]\right| \\
= & 1 .
\end{aligned}
$$

Apart from the trivial case $\lambda=0$ (isolated spins) this is possible if and only if $\cos \left(\mu_{0} T\right)=1$, that is, if $\mu_{0} T=2 \pi \mu_{0} / \omega$ is a multiple of $2 \pi$. This confirms the condition (51) independently derived from the quasienergy spectrum which in this case collapses to $\varepsilon_{0}=0$. Numerical analysis of the return probability $\left|u_{s s}(T)\right|$ for some arbitrarily chosen sender positions $s$ in chains of varying length led to the same result. Hence the system moves in synchronization with the drive and time-translation symmetry is not broken here.

Next, we investigate the possibility of breaking timetranslation symmetry by a $2 T$ periodicity. The matrix elements of the time evolution operator $U(2 T)$ can be obtained from those of $U(T)$ in 36

$$
\begin{aligned}
u_{r s}(2 T) & =\langle r|U(2 T)| s\rangle \\
& =\sum_{k=0}^{N} u_{r k}(T) u_{k s}(T) \\
& =e^{-i \mu_{0} N 2 T} \sqrt{\left(\begin{array}{c}
N \\
r
\end{array}\right)\left(\begin{array}{c}
N \\
s
\end{array}\right)} \\
& \times\left[\frac{4 \sqrt{p_{+} p_{-}}(1-\Gamma)\left(\Gamma+2 p_{+} p_{-}(1-\Gamma)^{2}\right)}{\Gamma^{2}+8 p_{+} p_{-}(1-\Gamma)^{2} \Gamma+8 p_{+}^{2} p_{-}^{2}(1-\Gamma)^{4}}\right]^{r+s} \\
& \times\left[\Gamma^{2}+8 p_{+} p_{-}(1-\Gamma)^{2} \Gamma+8 p_{+}^{2} p_{-}^{2}(1-\Gamma)^{4}\right]^{N} \\
& \times\left[1-p_{-}+p_{-} \Gamma\right]^{r}\left[1-p_{+}+p_{+} \Gamma\right]^{s} \\
& \times{ }_{2} F_{1}\left[\begin{array}{c}
(-r,-s) \\
(-N)
\end{array} \Upsilon \Upsilon\right.
\end{aligned}
$$

with

$$
\begin{aligned}
\Upsilon: & =-\frac{1}{16} \frac{\Gamma^{4}}{\left(\Gamma+2 p_{+} p_{-}(1-\Gamma)^{2}\right]^{2}} \\
& \times \frac{1}{p_{+} p_{-}(1-\Gamma)^{2} \Gamma+p_{+}^{2} p_{-}^{2}(1-\Gamma)^{4}} .
\end{aligned}
$$

Details of the calculation can be found in appendix B. Using sum and product formulas for hypergeometric functions, similar formulas for higher multiples of $T$ can be derived in increasingly tedious ways. The periodicity condition for period $2 T$ can be obtained in a way analogous to (57). $\left|u_{00}(2 T)\right|=1$ is equivalent to the condition

$$
\left|\Gamma^{2}+8 p_{+} p_{-}(1-\Gamma)^{2} \Gamma+8 p_{+}^{2} p_{-}^{2}(1-\Gamma)^{4}\right|^{2}=1
$$

which is in turn equivalent to

$$
\begin{aligned}
& \left|-2 c^{2}\left(1-\cos \left(\mu_{0} T\right)\right)^{2}+4 c\left(1-\cos \left(\mu_{0} T\right)\right)-1\right| \\
& =:\left|f_{c}\left[1-\cos \left(\mu_{0} T\right)\right]\right| \\
& =1 \\
& \text { with } c:=\lambda^{2} /\left(h^{2}+\lambda^{2}\right) \in(0,1) .
\end{aligned}
$$

The function $f_{c}(y)$ is quadratic in the variable $y:=$ $\left(1-\cos \left(\mu_{0} T\right)\right)$ taking values in the interval $0 \leq y \leq 2$. The value $f_{c}(y)=1$ which satisfies $(61)$, is reached as a maximum of $f_{c}(y)$ at $y=1 / c$, for $c>1 / 2$, that is, $h<\lambda$. 
In this case 61 is fulfilled by

$$
\cos \left(\mu_{0} T\right)=1-\frac{1}{c}=-\frac{h^{2}}{\lambda^{2}}
$$

implying

$$
\mu_{0} T=\pi \pm \delta, \quad 0 \leq \delta \leq \frac{\pi}{2}
$$

The condition 62 is incompatible with the condition 57) for $T$-periodic behavior, hence the observed period $2 T$ is not a trivial consequence of $T$-periodic dynamics.

Another possibility to fulfill (61) is

$$
f_{c}(y)=-1 \text {. }
$$

Obviously, that is true for $y=0$, but in that case 57 holds and the true period of the dynamics is $T$, not $2 T$. The only other possibility to fulfill $(64)$ occurs at $y=2$, with $c=1$, but that implies $h=0$ so that there is no drive at all.

In this subsection we have studied the breaking of timetranslation symmetry in the binarily driven system described by the Hamiltonian (32) from two perspectives. We derived a closed-form expression for the matrix elements of the propagator $U(T), \sqrt{36})$, which contains the complete information about the dynamics of arbitrary initial states. For arbitrary $N$, reducing this wealth of information to the eigenvalues of $U(T)$, or the equivalent quasienergy spectrum, is only possible numerically. Luckily the $S U(2)$ structure implied by the large spin form 31 of the Hamiltonian allows for the determination of the quasienergy spectrum in the $N=1$ case. It turns out that judiciously picking the parameters $\lambda$ and $h$ from a moderate region 52 of the $(\lambda, \omega)$ plane suffices to adjust the quasienergies such that the driven system breaks time-translation symmetry by being periodic with an arbitrary multiple, $n T$, of the driving period. As an example, time-translation symmetry breaking was independently analyzed by constructing the full matrix representation of $U(2 T)$ (59) and deriving conditions under which $U(2 T)$ (but not $U(T)$ ) equals unity.

We add some brief comments on perfect state transfer, the task for which the undriven $(h=0)$ system was originally 21 designed, as mentioned in Sec. IV A We found that driven systems satisfying the resonance condition (11) exhibit perfect state transfer at time $n T / 2$, reflecting the periodicity $n T$ combined with spatial symmetry. For the case $n=2$ this numerical observation can again be confirmed by analyzing the propagator $U(T)$. Setting $m_{0}=0$ in (53) leads to $T \mu_{0}=\pi$ for the driving period. If we set $\varphi=\pi / 4$ in $(55)$, equivalent to $c=1 / 2$ in 61 we obtain $\varepsilon_{0}=1 / 2 \omega / 2$, that is, $n=2$ in (11). We then see that $\left|u_{0 N}(T)\right|=1$ is equivalent to

$$
\left|\left[2 \sqrt{p_{+} p_{-}}(1-\Gamma)\right]^{N}\left[1-p_{-}+\Gamma p_{-}\right]^{N}\right|=1
$$

which leads to

$$
\left|\left[4 \sqrt{p_{+} p_{-}}\right]\left[1-2 p_{-}\right]\right|=\left|\left[\frac{4}{\sqrt{8}}\right]\left[\frac{1}{\sqrt{2}}\right]\right|=1 .
$$

We thus have periodicity $2 T$ and perfect state transfer between sites 0 and $N$ at time $T$. Keeping in mind that arbitrary periods $n T$ can be reached by adjusting $\lambda$ and $h$, it is clear that perfect state transfer can be slowed down arbitrarily by suitably driving the system. However, it cannot be accelerated by the binary drive, as a closer look at the time and frequency relations reveals.

\section{Harmonic drive}

The results obtained in Sec. IV B are not specific to the binary type of drive. As long as the choice 21) of nearestneighbor couplings and local fields is not changed, the Hamiltonian is still equivalent to the large spin model (31) with time-dependent $\lambda$ and $h$, implying that the time evolution operator $U(T)$ is an element of the group $S U(2)$. Different driving protocols then always yield the same structure (24) of equidistant quasienergies and time-translation symmetry breaking can be reached by adjusting the parameters of the drive.

As an example, we investigated the harmonic drive defined by

$$
f(t)=2 \cos (\omega t)
$$

in the Hamiltonian (32). Using the Floquet matrix formalism introduced in Sec. III the quasienergy spectrum was determined, with the fundamental quasienergy unit $\varepsilon_{0}$ depending on $\lambda$ and $h$ in a qualitatively similar way as displayed in Fig. 3 for binary driving. For the harmonic drive, however, the shape of the ridges and valleys in the $(\lambda, h)$-plane is approximately elliptic $\frac{48}{\text {, }}$, as opposed to circular as in Fig. 3. Due to these structural similarities, the phenomena from Sec. IV B reappear: periodicity with the period $T$ of the drive and with arbitrary multiples $n T$ of it. (See Fig. $4 \mathrm{~d}$ below for a case with $n=3$.) However, one difference to the binarily driven case arises. For the harmonic drive, periodically driven perfect state transfer is possible as it is for the binary drive, but only if $m$ and $n$ are both odd in the resonance condition (11), which can be achieved by adjusting $\lambda$ and $h$. In fact, similar results are obtained for fairly general combinations of the parameters $\lambda$ and $h$. It is even possible to exchange the roles of the driven and constant parts of the Hamiltonian $(32)$, that is, harmonically driving the nearest-neighbour interactions $J_{i}(t)$ while keeping the local potentials $h_{i}(t)$ constant. Employing the large spin picture (31) that change is nothing but a switching of roles between the spin operators $S_{x}$ and $S_{z}$.

\section{Robustness and heating}

We have seen that discrete time-translation symmetry can be broken in the driven spin chain studied here. To achieve that, the parameters $\lambda$ and $h$ of the Hamiltonian must be adjusted. In order to learn about the stability of 
the periodic phase we studied the system with additional spatial disorder. Admittedly there are also many other kinds of perturbation to which the system might be subjected. Our specific choice of perturbation is motivated by the role of the spin chain in the field of quantum information transfer, where robustness against manufacturing errors is an issue of interest $\frac{46}{}$.

We have considered systems in which the coefficients (21) are perturbed by disorder

$$
J_{i} \longrightarrow J_{i}\left(1+x_{i}\right)
$$

(and similar for $h_{i}$ ), where the $x_{i}$ are independent identically distributed random variables drawn from a Gaussian distribution with mean zero and standard deviation $\sigma$. In Fig. 4 we show results for independently varying standard deviations of the $J_{i}$ and $h_{i}$, denoted by $\sigma_{\lambda}$ and $\sigma_{h}$, respectively. Shown is the the averaged absolute value of the return amplitude $f_{s, s}\left(m T_{S}\right):=\left\langle s\left|U\left(m T_{S}\right)\right| s\right\rangle$ as a function of $\sigma_{h}$ and $\sigma_{\lambda}$. As usual, $T_{S}=n T$ denotes the period of the system as a multiple of the driving period and the return amplitude is determined after $m$ periods.

As an example we explain the choice of parameters in Figs. $4 \mathrm{a}$ and $4 \mathrm{~b}$.

$$
\lambda=0.3 \text { and } h=0.273316
$$

leads to $\mu_{0}=\sqrt{\lambda^{2}+h^{2}}=0.405834$. The driving period $T=2 \pi(\omega=1)$ then leads to

$$
h^{2}+\lambda^{2} \cos \mu_{0} T=0
$$

from which by 38

$$
\varepsilon_{0}=\frac{1}{2 \pi} \arccos (0)=\frac{1}{4},
$$

hence the resonance condition (11) is fulfilled with $m=1$ and $n=2$ such that we have time-translation symmetry breaking with period

$$
T_{S}=2 T=4 \pi
$$

(Note that this choice of $\varepsilon_{0}$ seems to violate conditions (9), but those conditions are merely sufficient and not necessary.)

Figs. $4 \mathrm{a}$ and $4 \mathrm{~b}$ refer to the same binarily driven system with $T_{S}=2 T$, after one period and three periods, respectively. There is a region of high fidelity $f_{s, s}$ around the unperturbed case. However, that region shrinks as time grows. Figs. 4c and 4d compare different systems with $T_{S}=3 T$, after one period. One system is driven binarily, the other one harmonically. The behavior of $f_{s, s}$ is roughly similar in both cases.

The results displayed in Fig. 4 show that the timetranslation symmetry breaking in our system is robust against disorder for short times. We have also performed numerical analyses of robustness on longer time scales, and for different system sizes $N$. In this context it must be noted that, strictly speaking, our system does not have a thermodynamic limit in the conventional sense, since the Hamiltonian parameters (21) depend on $N$. Hence the criterion (1) involving long-range order in the thermodynamic limit can only be applied to the present model for finite, but large system size; we analyzed systems with $N \leq 110$. The local quantity $C(x, t)$ from (1) is the probability amplitude $C(n, t)=f_{0 n}(t)$ for a localized spin excitation to reach site $n$ of the chain at time $t$ after starting from site 0 at time 0 . Since we want to demonstrate time-crystalline behavior at long distance in space we consider the quantity

$$
\left\langle f_{0 N}(t) f_{00}(0)\right\rangle=\left\langle f_{0 N}(t)\right\rangle .
$$

(Note that $f_{00}(0)=1$.) This quantity was calculated for systems with Hamiltonian parameters 69 subjected to multiplicative disorder (68), and also to additive disorder, defined by

$$
J_{i} \longrightarrow J_{i}+x_{i}
$$

(and similar for $h_{i}$ ), with Gaussian $x_{i}$, as before. We have also simulated $x_{i}$ from a uniform distribution of finite width, but the results are similar to those for Gaussian $x_{i}$ and are not shown here. Note that due to the $N$ dependence of the unperturbed Hamiltonian parameters $J_{i}$ and $h_{i}$ the two types of disorder are expected to affect the system in different ways as the system size changes. This is indeed so, as the numerical results discussed below show.

In the absence of disorder the correlation function $\left\langle f_{0 N}(t)\right\rangle$ is (of course) perfectly periodic and shows Gaussian-looking peaks of unit height and width (in time) proportional to $N^{-\frac{1}{2}[51}$. The Fourier series of $\left\langle f_{0 N}(t)\right\rangle$ then has Fourier coefficients which also scale as $N^{-1 / 2}$ for sufficiently large $N$.

In the presence of disorder, successive peaks of $\left\langle f_{0 N}(t)\right\rangle$ get lower and wider. For given strength $\sigma$ of the disorder the dependence on system size differs considerably between the two types of disorder considered. For additive disorder the peaks of the correlation function are the more stable the longer the chains are while for multiplicative disorder shorter chains are more stable. Since the $J_{i}$ and $h_{i}$ grow with $N$, a random perturbation of constant absolute size becomes less and less important as $N$ grows, even though the total number of perturbed coupling constants grows. In contrast, multiplicative disorder of a given strength is much more detrimental since it generates a larger number of larger absolute deviations of the $J_{i}$ and $h_{i}$ from their ideal values as $N$ grows. This effect is clearly visible in Fig. 5, see below.

As $\left\langle f_{0 N}(t)\right\rangle$ changes from perfectly periodic to slowly decaying, its Fourier transform changes from equidistant $\delta$ function peaks to equidistant finite peaks. The spectral weight contained in the peak at the lowest nonzero frequency has been considered a good indicator of time crystallinity $7 / 9|12| 15 \mid 17$. Fig. 5 shows results for that spectral weight in the presence of disorder, for two types and 


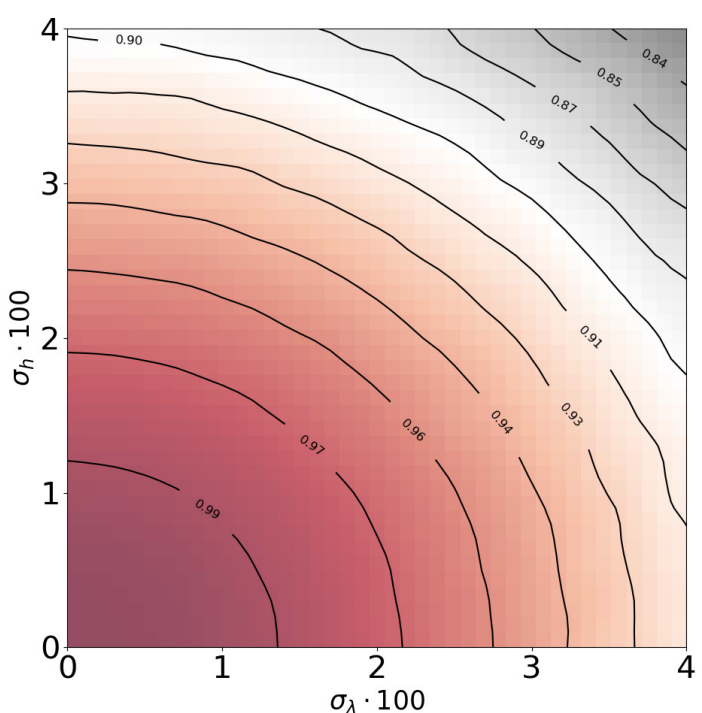

(a) Binary drive; $n=2$ and $m=1$.

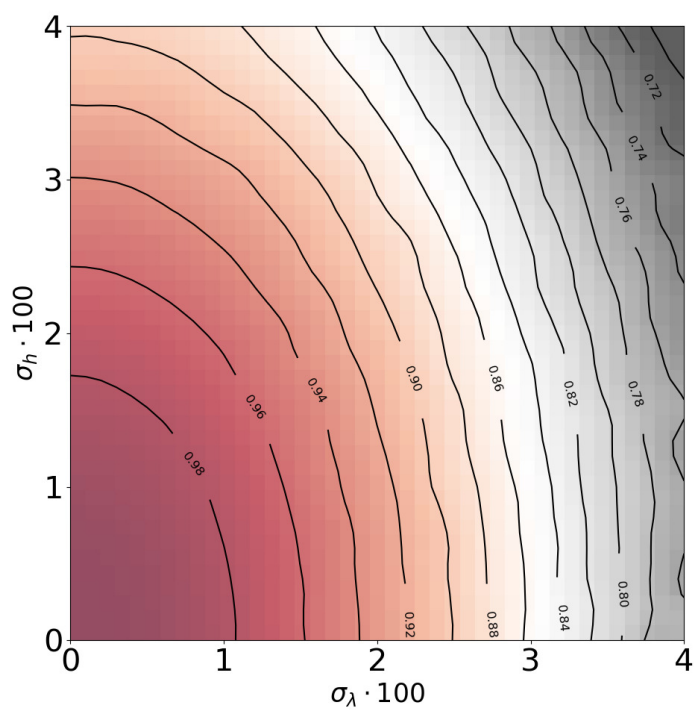

(c) Binary drive; $n=3$ and $m=1$.
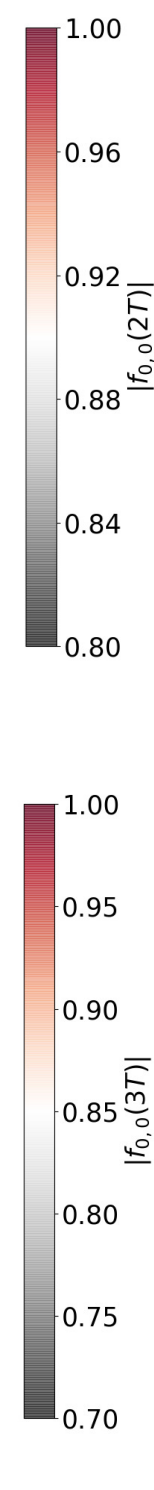

0.95

0.90

0.80

0.75

70

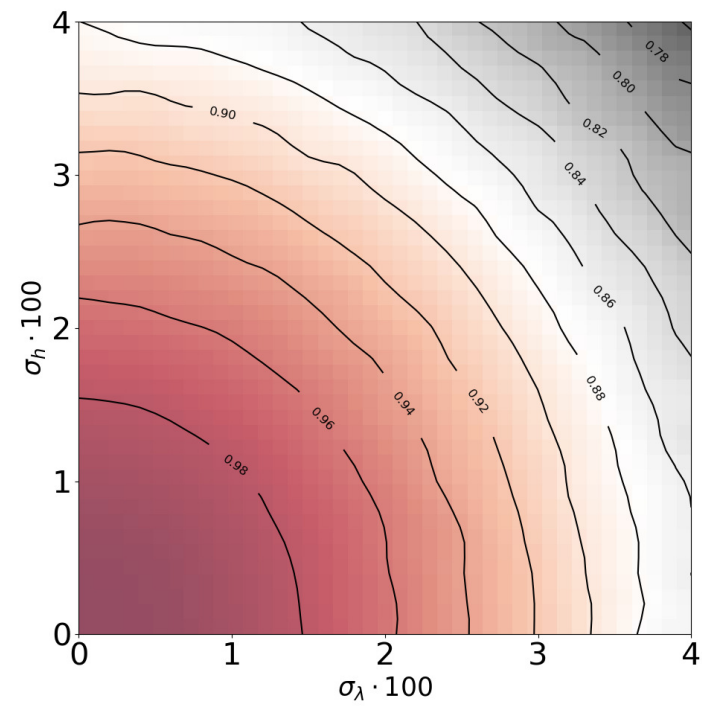

(d) Harmonic drive; $n=3$ and $m=1$.

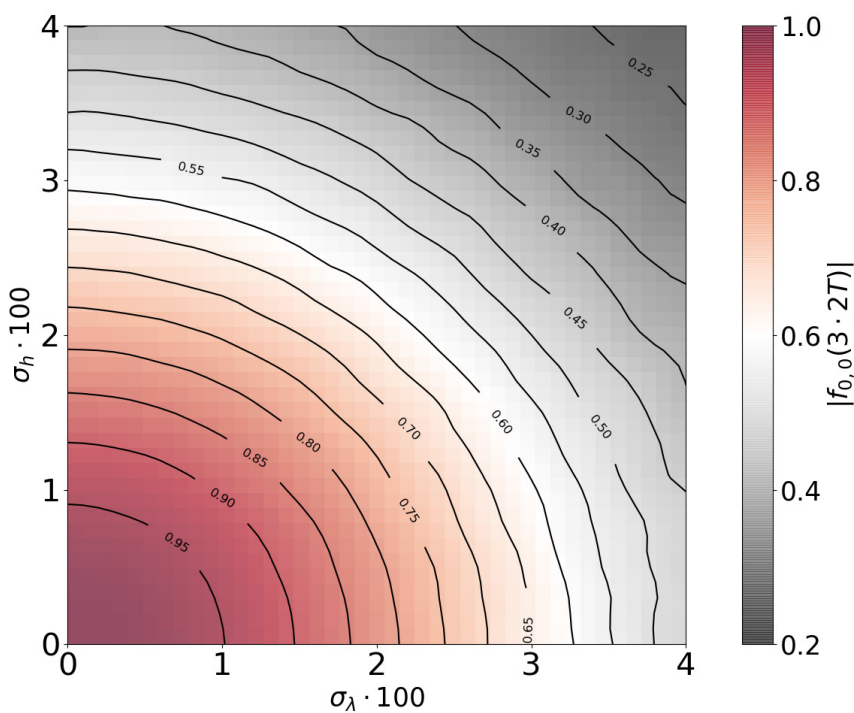

(b) Binary drive; $n=2$ and $m=3$.

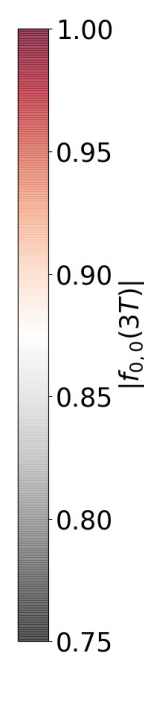

FIG. 4: Stability of the time-translation symmetry breaking against spatial randomness in the system. Shown is the absolute value of the return amplitude $f_{0,0}=\left\langle 0\left|U\left(m T_{S}\right)\right| 0\right\rangle$ in the system with multiplicative Gaussian disorder (68) in the Hamiltonian, as a function of the standard deviations $\sigma_{h}$ and $\sigma_{\lambda}$. Time-translation symmetry is broken with $T_{S}=2 T$ in panels (a) and (b), and with $T_{S}=3 T$ in panels (c) and (d), respectively. Panel (d) shows results for harmonic driving, all other results are for binary driving. In all cases, $N=29$ and $\omega=1$; all data were averaged over 640 measurements. A Gaussian smoothing was applied to the fluctuating raw data in order to avoid wildly fluctuating contour lines. Parameters used were $\lambda=0.3, h=0.273316$ in panels (a) and (b), $\lambda=0.2, h=0.304237$ in panel (c), and $\lambda=1.2, h=1.279452$ in panel $(\mathrm{d})$.

two strengths of disorder, as a function of system size. In order to eliminate the intrinsic (disorder-independent) size dependence, the peak heights of the disordered systems are divided by the peak heights of the ordered systems of equal size. The figure shows that time-translation symmetry breaking is affected by static disorder in the local parameters $J_{i}$ and $h_{i}$. It is, however, still robust on the intermediate time scale studied here, the degree of robustness depending on the type and strength of disorder.
Note that due to the particular scaling of the Hamiltonian with $N$ the system does not possess a thermodynamic limit in the strict sense. However, since our model is equivalent to a single-particle system, we can treat much larger system sizes than other studies $7 / 9|12| 15$.

One general issue in driven systems is heating. We solved the driven model under study exactly by determining its quasi-energies and computing spatio-temporal correlations. Time-crystalline behavior occurs if and only if 


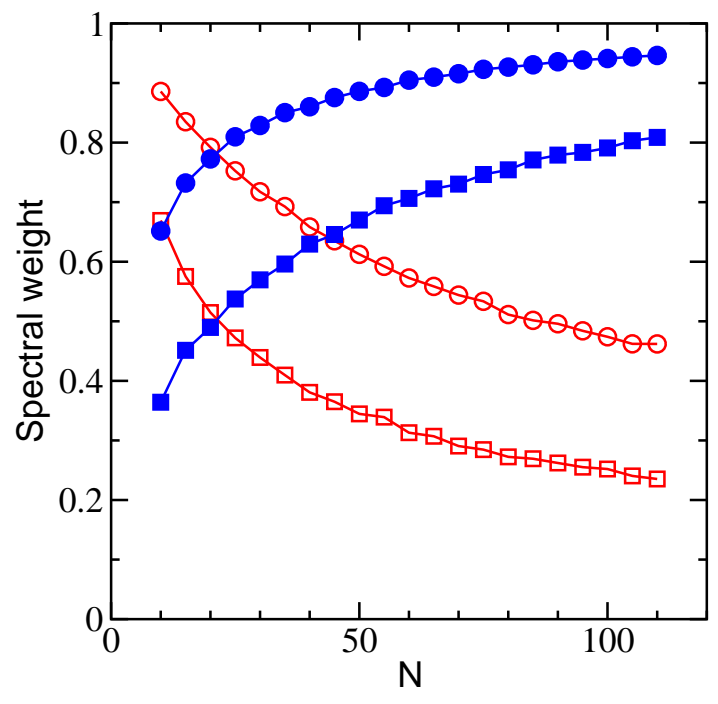

FIG. 5: Spectral weight $w\left(\frac{\omega}{2}\right)$ of the fundamental time crystal mode, for different kinds of disorder, as a function of system size. The time correlation $\left\langle f_{0 N}(t)\right\rangle$ was calculated for $4000 t$ values between zero and $8 T_{S}$, averaging over 500 disordered system configurations. The modulus-squared of the (numerical) Fourier transform of $\left\langle f_{0 N}(t)\right\rangle$ defines the spectral weight. The height of the peak at $\frac{\omega}{2}$ (where $\omega$ is the driving frequency) of that quantity is divided by the same quantity, calculated without disorder, to eliminate the intrinsic (disorder-independent) size dependence. All data shown are for Gaussian disorder. Types of disorder are multiplicative, for $\sigma=0.01$ (open red circles) and $\sigma=0.02$ (open red squares) as well as additive, for $\sigma=0.1$ (filled blue circles) and $\sigma=0.2$ (filled blue squares).

the quasi-energies are multiples of a fundamental energy, thus they form a discrete, equidistant spectrum without continua. No heating occurs. This is perfectly consistent with the general understanding of energy flow known from Fermi's Golden Rule: a continued flow of energy into a system requires a continuum of energies. If there are only discrete states energy flows back and forth and no net heating takes place. Hence, we understand that the absence of heating is not a particularity of the model studied, but a particularity of a system which shows timecrystalline behavior for all initial states. Any modification of the model which leads to quasi-energy continua destroys the time-crystalline behavior, but it may allow for heating. Thus, we stress that time-crystalline behavior and heating are mutually exclusive.

\section{CONCLUDING REMARKS}

Time crystals and the associated broken time translation symmetry have attracted a lot of interest during the past few years, generating a new perspective towards driven systems. To what extent interaction and/or disorder are necessary ingredients for time-crystalline behavior has been subject of a lively discussion. A number of model systems were studied numerically and displayed discrete time-crystalline behavior, mostly for specific initial states and for specific multiples of the driving period. Here, we studied a spatially extended driven single-particle system which shows breaking of discrete time-translation symmetry for arbitrary initial states and with arbitrary period controlled by adjusting two Hamiltonian parameters.

The system is a spin- $1 / 2$ chain originally designed as a candidate for the realization of perfect quantum state transfer in a one-dimensional system, also motivating investigations into the influence of an external periodic drive. Without driving, the system shows an equidistant spectrum of energy eigenvalues, implying periodic behavior in time and thus recurrence of arbitrary initial states. We generalized this property of the engineered spin chain to the driven version of the system.

Under the influence of external periodic driving, Floquet theory can be applied, and the relevant quantities are no longer the energies, but the quasienergies. Similar to the time independent case, it turns out that the spectrum exhibits an equidistant integer spectrum of quasienergies with some smallest "quasienergy quantum" $\varepsilon_{0}$ related to the parameters of the system in a nontrivial way.

Based on this observation, some general conclusions about the dynamics are drawn. One crucial feature of the investigated system is the time translation symmetry breaking for all initial states which is not satisfied in many of the previously suggested time-crystalline systems. Periodicity for arbitrary multiples of the driving period can be achieved in a tunable manner. This possibility is not only appealing from a theoretical point of view, but also for possible experimental applications. Adjusting a small number of parameters (basically the driving strength) opens up the possibility to break discrete time-translation symmetry in a controlled way. We discussed this behavior for both a binary drive, where a system parameter is periodically switched discontinuously and for a harmonic drive with sinusoidal change of the same parameter. Numerical calculations show that the time-translational symmetry breaking persists in the presence of built-in static randomness in the system, for intermediate time scales and at distances up to about 100 lattice spacings.

Considering time-crystallization we re-iterate that there is so far no generally established definition for a time crystal. The studied model fulfills a number of crucial criteria: breaking of time translational invariance, occurrence of subharmonic dynamics for all initial states 
for arbitrary system size, and a certain robustness: the spectral weight of the subharmonics is decreased only gradually by disorder. Certain other criteria which are discussed to be important are not met: the system does not have a thermodynamic limit, it is a single-particle problem after suitable mapping, and time translational symmetry breaking is not robust in the sense that the subharmonics remain unchanged up to a certain threshold for the variation of parameters. Whether the latter criteria can be met by any physical system remains an open point to date.

The effects reported here have potentially useful applications in quantum information processing. The nearest neighbor coupling constants along the spin chain may be fixed once and for all. Then, the periodicity of the system could be modified by tuning strength and frequency of the periodically varying external field. This can be used for transfer of a quantum state at a prescribed time or for a dynamic memory which allows for the readout of an initial state whenever it refocuses.

\section{ACKNOWLEDGMENTS}

We gratefully acknowledge helpful discussions with David J. Luitz (R.S.), Susan Coppersmith, and Oleg P. Sushkov (G.S.U). This research was supported by the Deutsche Forschungsgemeinschaft (Grant No. UH 9013/1) (G.S.U.). Furthermore, the manuscript was completed at the School of Physics of the University of New South Wales and G.S.U. acknowledges its hospitality and the Heinrich-Hertz Foundation for financial support of this visit.

\section{Appendix A: Krawtchouk polynomials}

The Krawtchouk polynomials $K_{n}^{p}(x)$ are based on the hypergeometric series

$$
{ }_{r} F_{s}\left[\begin{array}{c}
\left(a_{1}, \ldots, a_{r}\right) \\
\left(b_{1}, \ldots, b_{s}\right)
\end{array} ; z\right]:=\sum_{k=0}^{\infty} \frac{\left(a_{1}\right)_{k}\left(a_{2}\right)_{k} \ldots\left(a_{r}\right)_{k}}{\left(b_{1}\right)_{k}\left(b_{2}\right)_{k} \ldots\left(b_{s}\right)_{k}} \frac{z^{k}}{k !} \text { with }(x)_{k}:=\left\{\begin{array}{ll}
1 & \text { for } k=0 \\
x(x+1) \ldots(x+k-1) & \text { for } k \geq 1
\end{array} .\right.
$$

$(x)_{k}$ is known as the Pochhammer symbol. For given $N$ and $0<p<1$ a set of discrete polynomials, labeled by

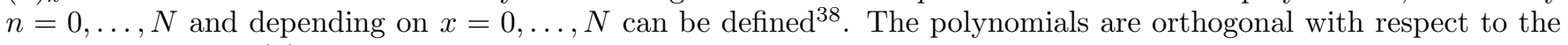
weight function $w^{p}(x)$ and the normalization constant $d_{n}^{p}$ :

$$
\begin{aligned}
& K_{n}^{p}(x):={ }_{2} F_{1}\left[\begin{array}{c}
(-x,-n) \\
(-N)
\end{array} ; \frac{1}{p}\right], \text { for } x=0, \ldots, N \\
& \text { with } w^{p}(x):=\left(\begin{array}{c}
N \\
x
\end{array}\right) p^{x}(1-p)^{N-x}, \quad d_{n}^{p}:=\left(\begin{array}{c}
N \\
n
\end{array}\right)^{-1}\left(\frac{1-p}{p}\right)^{n} \\
& \\
& \Rightarrow \sum_{x=0}^{N} w^{p}(x) K_{n}^{p}(x) K_{m}^{p}(x)=d_{n}^{p} \delta_{n m} .
\end{aligned}
$$

Note that $F(-x,-n,-N, 1 / p)=0$ for $x, n>N$; therefore, each term of the series in A1) is zero for $x, n>N$. We refer to the orthogonal and normalized Krawtchouk polynomials

$$
\kappa_{n}^{p}(x):=\sqrt{\frac{w^{p}(x)}{d_{n}^{p}(x)}} K_{n}^{p}(x) .
$$

The spin system suggested by Christandl et al.21 and generalized as discussed in Sec IV A can be solved using the recurrence relation of the Krawtchouk polynomials:

$$
\begin{aligned}
-x K_{n}^{p}(x) & =n(1-p) K_{n-1}^{p}(x)-[p(N-n)+n(1-p)] K_{n}^{p}(x)+(N-n) p K_{n+1}^{p}(x) \\
\Rightarrow-x \kappa_{n}^{p}(x) & =\sqrt{p(1-p)} J_{n-1} \kappa_{n-1}^{p}(x)-[p(N-n)+n(1-p)] \kappa_{n}^{p}(x)+\sqrt{p(1-p)} J_{n} \kappa_{n+1}^{p}(x) .
\end{aligned}
$$

$p \in(0,1)$ has to be adjusted according to 22 to diagonalize the Hamiltonian. Relations including hypergeometric

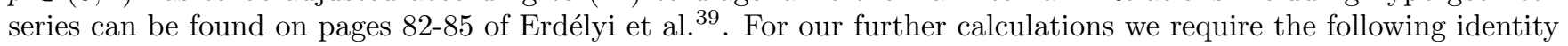

$$
\begin{aligned}
& \sum_{n=0}^{N}\left(\begin{array}{l}
N \\
n
\end{array}\right) s^{n}{ }_{2} F_{1}\left[\begin{array}{c}
(-n,-b) \\
(-N)
\end{array} ; z\right]{ }_{2} F_{1}\left[\begin{array}{c}
(-n,-\beta) \\
(-N)
\end{array} ; \zeta\right] \\
& =(1+s)^{N-b-\beta}(1+s-s z)^{b}(1+s-s \zeta)_{2}^{\beta} F_{1}\left[\begin{array}{c}
(-b,-\beta) \\
(-N)
\end{array} ; \frac{-s z \zeta}{(1+s-s z)(1+s-s \zeta)}\right] .
\end{aligned}
$$


Inserting the appropriate $p$, the transmission amplitudes 27) can be derived for the undriven system (Sec IV A):

$$
\begin{aligned}
& f_{r s}^{p}(t)=\left\langle r\left|e^{-i H t}\right| s\right\rangle=\left\langle r\left|\sum_{x=0}^{N} e^{-i t E_{x}}\right| \varphi_{x}\right\rangle\left\langle\varphi_{x}|| s\right\rangle=\sum_{x=0}^{N} \kappa_{r}^{p}(x)^{\dagger} \kappa_{s}^{p}(x) e^{-i t E_{x}}=\frac{1}{\sqrt{d_{r}^{p} d_{l}^{p}}} \sum_{x=0}^{N} w^{p}(x) K_{r}^{p}(x) K_{s}^{p}(x) e^{-i t E_{x}} \\
& =\frac{e^{-i t \mu_{0} N}(1-p)^{N}}{\sqrt{d_{r}^{p} d_{l}^{p}}} \sum_{x=0}^{N}\left(\begin{array}{c}
N \\
x
\end{array}\right)\left[\frac{p}{1-p} \Gamma\right]_{2}^{x} F_{1}\left[\begin{array}{c}
(-x,-r) \\
(-N)
\end{array} ; \frac{1}{p}\right]{ }_{2} F_{1}\left[\begin{array}{c}
(-x,-s) \\
(-N)
\end{array} ; \frac{1}{p}\right] \\
& \underline{\text { A6 }}=\frac{e^{-i t \mu_{0} N}(1-p)^{N}}{\sqrt{d_{r}^{p} d_{l}^{p}}}\left[\frac{1-p+p \Gamma}{1-p}\right]^{N-r-s}\left[\frac{1-p+p \Gamma-\Gamma}{1-p}\right]^{r+s}{ }_{2} F_{1}\left[\begin{array}{c}
(-r,-s) \\
(-N)
\end{array} ;-\frac{\Gamma}{p(1-p)(1-\Gamma)^{2}}\right] \\
& =e^{-i t \mu_{0} N} \sqrt{\left(\begin{array}{c}
N \\
r
\end{array}\right)\left(\begin{array}{c}
N \\
s
\end{array}\right)}[\sqrt{p(1-p)}]^{r+s}[1-\Gamma]^{r+s}[1-p+p \Gamma]^{N-r-s}{ }_{2} F_{1}\left[\begin{array}{c}
(-r,-s) \\
(-N)
\end{array} ;-\frac{\Gamma}{p(1-p)(1-\Gamma)^{2}}\right] \text {, }
\end{aligned}
$$

with $\mu_{0}=\sqrt{\lambda^{2}+h^{2}}$ and $\Gamma=e^{i t 2 \mu_{0}}$. These results were already obtained by Van der Jeugt et al! $36 \mid 37$.

\section{Appendix B: Binary drive}

Using the transmission coefficients A7 derived in appendix A, the evolution operator and its matrix elements can be calculated using the product formula $\mathrm{A} 6$ for a binary drive. The system is stationary within each half period $T / 2$. First, $p_{-}$covers the case of linearly decreasing fields over $[0, T / 2$ ) (see Sec. IV B). The coefficients determined by $p_{+}$, linearly increasing fields, are used within $[T / 2, T)$.

The calculation of the coefficients is more complex than in the stationary case treated in appendix A. The following variables are introduced:

$$
\begin{aligned}
\zeta & :=-\frac{\Gamma}{p_{ \pm}\left(1-p_{ \pm}\right)(1-\Gamma)^{2}}=-\frac{\Gamma}{p_{+} p_{-}(1-\Gamma)^{2}}, \\
\theta_{r s} & :=e^{-i \mu_{0} N T} \sqrt{\left(\begin{array}{c}
N \\
r
\end{array}\right)\left(\begin{array}{c}
N \\
s
\end{array}\right)}\left[\sqrt{p_{+} p_{-}}\right]^{r+s}[1-\Gamma]^{r+s}\left[1-p_{-}+p_{-} \Gamma\right]^{N-s}\left[1-p_{+}+p_{+} \Gamma\right]^{N-r}, \\
\phi_{r s}: & =e^{-i \mu_{0} N 2 T} \sqrt{\left(\begin{array}{c}
N \\
r
\end{array}\right)\left(\begin{array}{c}
N \\
s
\end{array}\right)}\left[1-p_{-}+p_{-} \Gamma\right]^{r}\left[1-p_{+}+p_{+} \Gamma\right]^{s}\left[\frac{2 \sqrt{p_{+} p_{-}}(1-\Gamma)}{\Gamma+2 p_{+} p_{-}(1-\Gamma)^{2}}\right]^{r+s}\left[\Gamma+2 p_{+} p_{-}(1-\Gamma)^{2}\right]^{2 N},
\end{aligned}
$$

with $\Gamma=e^{i T \mu_{0}}$. Additionally, some relations involving $p_{ \pm}$are needed:

$$
\begin{aligned}
p_{ \pm}=\frac{1}{2} \pm \frac{1}{2} \sqrt{1-\frac{\lambda^{2}}{\lambda^{2}+h^{2}}} \Rightarrow & p_{ \pm}\left(1-p_{ \pm}\right)=p_{+} p_{-}=\frac{1}{4} \frac{\lambda^{2}}{\lambda^{2}+h^{2}}, \quad p_{+}+p_{-}=1 \\
& \text { and }\left(1-p_{-}+p_{-} \Gamma\right)\left(1-p_{+}+p_{+} \Gamma\right)=\Gamma+p_{+} p_{-}(1-\Gamma)^{2}
\end{aligned}
$$

The derivation of $u_{r s}(T), 36$ then proceeds as follows:

$$
\begin{aligned}
& u_{r s}(T)=\langle r|U(T)| s\rangle=\sum_{k=0}^{N} f_{r k}^{p_{+}}(T / 2) f_{k s}^{p_{-}}(T / 2) \\
& \quad \stackrel{p_{+} p_{-}(1-\Gamma)^{2}}{\Gamma} \theta_{r s} \sum_{k=0}^{N}\left(\begin{array}{l}
N \\
k
\end{array}\right)\left[{ }_{2} F_{1}\left[\begin{array}{c}
(-r,-k) \\
(-N)
\end{array} ; \zeta\right]{ }_{2} F_{1}\left[\begin{array}{c}
(-k,-s) \\
(-N)
\end{array} ; \zeta\right]\right.
\end{aligned}
$$


We apply the summation formula $\mathrm{A} 6$. The following terms occur:

$$
\begin{array}{rlrl}
s & :=\frac{p_{+} p_{-}(1-\Gamma)^{2}}{\Gamma+p_{+} p_{-}(1-\Gamma)^{2}} \\
\Rightarrow \quad 1+s & =\frac{\Gamma+2 p_{+} p_{-}(1-\Gamma)^{2}}{\Gamma+p_{+} p_{-}(1-\Gamma)^{2}} \\
\Rightarrow \quad 1+s-s \zeta \stackrel{\text { B } 1 / 2}{-} \frac{\Gamma+2 p_{+} p_{-}(1-\Gamma)^{2}}{\Gamma+p_{+} p_{-}(1-\Gamma)^{2}}-\frac{p_{+} p_{-}(1-\Gamma)^{2}}{\Gamma+p_{+} p_{-}(1-\Gamma)^{2}} \frac{(-1) \Gamma}{p_{+} p_{-}(1-\Gamma)^{2}}=2 \\
\Rightarrow \quad \frac{\Gamma^{2}}{(1+s-s \zeta)^{2}}=-\frac{1}{4} \frac{p_{+} p_{-}(1-\Gamma)^{2}}{\Gamma+p_{+} p_{-}(1-\Gamma)^{2}} \frac{\Gamma^{2}}{\left(p_{+} p_{-}\right)^{2}(1-\Gamma)^{4}}=\underbrace{-\frac{1}{\Gamma+p_{+} p_{-}(1-\Gamma)^{2}} \frac{1}{p_{+} p_{-}(1-\Gamma)^{2}}}_{=: \eta}
\end{array}
$$

The determination of $u_{r s}(T)$ is possible using the previously derived relations:

$$
u_{r s}(T)=\theta_{r s}\left[\frac{\Gamma+2 p_{+} p_{-}(1-\Gamma)^{2}}{\Gamma+p_{+} p_{-}(1-\Gamma)^{2}}\right]^{N-r-s}[2]^{s+r}{ }_{2} F_{1}\left[\begin{array}{c}
(-r,-s) \\
(-N)
\end{array} ; \eta\right]
$$

Inserting $\theta_{r s}$ finally yields 36 .

Next, we derive $u_{r s}(2 T)$, (58), in a similar manner:

$$
\begin{aligned}
& u_{r s}(2 T)=\sum_{k=0}^{N} u_{r k}(T) u_{k s}(T) \\
& \stackrel{\text { B3 } 3}{=} \phi_{r s} \sum_{k=0}^{N}\left(\begin{array}{c}
N \\
k
\end{array}\right)\left[\frac{4 p_{+} p_{-}(1-\Gamma)^{2} \Gamma+4 p_{+}^{2} p_{-}^{2}(1-\Gamma)^{4}}{\Gamma^{2}+4 p_{+} p_{-}(1-\Gamma)^{2} \Gamma+4 p_{+}^{2} p_{-}^{2}(1-\Gamma)^{4}}\right]^{k}{ }_{2} F_{1}\left[\begin{array}{c}
(-r,-k) \\
(-N)
\end{array} ; \eta\right]{ }_{2} F_{1}\left[\begin{array}{c}
(-k,-s) \\
(-N)
\end{array} ; \eta\right] .
\end{aligned}
$$

Again, we apply the summation formula in A6. The following terms occur:

$$
\begin{aligned}
& \tilde{s}:=\frac{4 p_{+} p_{-}(1-\Gamma)^{2} \Gamma+4 p_{+}^{2} p_{-}^{2}(1-\Gamma)^{4}}{\left[\Gamma+2 p_{+} p_{-}(1-\Gamma)^{2}\right]^{2}} \\
& \Rightarrow \quad 1+\tilde{s}=\frac{\Gamma^{2}+8 p_{+} p_{-}(1-\Gamma)^{2} \Gamma+8 p_{+}^{2} p_{-}^{2}(1-\Gamma)^{4}}{\left[\Gamma+2 p_{+} p_{-}(1-\Gamma)^{2}\right]^{2}} \\
& \Rightarrow \quad 1+\tilde{s}-\tilde{s} \eta=1+\tilde{s}+\frac{\Gamma^{2}}{\left[\Gamma+2 p_{+} p_{-}(1-\Gamma)^{2}\right]^{2}}=2 \\
& \Rightarrow \quad \frac{-\tilde{s} \eta^{2}}{(1+\tilde{s}-\tilde{s} \eta)^{2}}=\underbrace{-\frac{1}{16} \frac{\Gamma^{4}}{\left[\Gamma+2 p_{+} p_{-}(1-\Gamma)^{2}\right]^{2}} \frac{1}{p_{+} p_{-}(1-\Gamma)^{2} \Gamma+p_{+}^{2} p_{-}^{2}(1-\Gamma)^{4}}}_{=: \Upsilon}
\end{aligned}
$$

The determination of $u_{r s}(2 T)$ is then possible using the previously derived relations:

$$
\begin{aligned}
& u_{r s}(2 T)=\phi_{r s} \sum_{k=0}^{N}\left(\begin{array}{c}
N \\
k
\end{array}\right)[\tilde{s}]_{2}^{k} F_{1}\left[\begin{array}{c}
(-r,-k) \\
(-N)
\end{array} ; \eta\right]{ }_{2} F_{1}\left[\begin{array}{c}
(-k,-s) \\
(-N)
\end{array} ; \eta\right] \\
& =\phi_{r s}\left[\frac{\Gamma^{2}+8 p_{+} p_{-}(1-\Gamma)^{2} \Gamma+8 p_{+}^{2} p_{-}^{2}(1-\Gamma)^{4}}{\left[\Gamma+2 p_{+} p_{-}(1-\Gamma)^{2}\right]^{2}}\right]^{N-r-s}[2]^{s+r}{ }_{2} F_{1}\left[\begin{array}{c}
(-r,-s) \\
(-N)
\end{array} ; \Upsilon\right] .
\end{aligned}
$$

\footnotetext{
* robin.schaefer@tu-dortmund.de, schaefer@pks.mpg.de $\quad \dagger$ goetz.uhrig@tu-dortmund.de
} 
‡ joachim.stolze@tu-dortmund.de

1 F. Wilczek, Phys. Rev. Lett. 109, 160401 (2012)

2 P. Bruno, Phys. Rev. Lett. 110, 118901 (2013).

3 P. Nozières, EPL (Europhysics Letters) 103, 57008 (2013)

4 P. Bruno, Phys. Rev. Lett. 111, 029301 (2013)

${ }^{5}$ H. Watanabe and M. Oshikawa, Phys. Rev. Lett. 114, 251603 (2015)

${ }^{\circ}$ A. Chandran and S. L. Sondhi, Phys. Rev. B 93, 174305 (2016).

V. Khemani, A. Lazarides, R. Moessner, and S. L. Sondhi, Phys. Rev. Lett. 116, 250401 (2016)

8 C. W. von Keyserlingk, V. Khemani, and S. L. Sondhi, Phys. Rev. B 94, 085112 (2016)

9 N. Y. Yao, A. C. Potter, I.-D. Potirniche, and A. Vishwanath, Phys. Rev. Lett. 118, 030401 (2017).

10 K. Sacha, Phys. Rev. A 91, 033617 (2015).

11 D. V. Else, B. Bauer, and C. Nayak, Phys. Rev. Lett. 117, $090402(2016)$

I2 J. Zhang, P. W. Hess, A. Kyprianidis, P. Becker, A. Lee, J. Smith, G. Pagano, I.-D. Potirniche, A. C. Potter, A. Vishwanath, N. Y. Yao, and C. Monroe, Nature 543, 217 (2017).

13 B. Huang, Y.-H. Wu, and W. V. Liu, Phys. Rev. Lett. 120, 110603 (2018)

${ }^{14}$ W. C. Yu, J. Tangpanitanon, A. W. Glaetzle, D. Jaksch, and D. G. Angelakis, PRA 99, 033618 (2019).

15 R. E. Barfknecht, S. E. Rasmussen, A. Foerster, and N. T. Zinner, Phys. Rev. B 99, 144304 (2019).

16 F. M. Gambetta, F. Carollo, M. Marcuzzi, J. P. Garrahan, and I. Lesanovsky, PRL 122, 015701 (2019).

17 S. Choi, J. Choi, R. Landig, G. Kucsko, H. Zhou, J. Isoya, F. Jelezko, S. Onoda, H. Sumiya, V. Khemani, C. von Keyserlingk, N. Y. Yao, E. Demler, and M. D. Lukin, Nature 543, 221 (2017).

18 V. Khemani, C. W. von Keyserlingk, and S. L. Sondhi, Phys. Rev. B 96, 115127 (2017)

19 D. H. Dunlap and V. M. Kenkre, Phys. Rev. B 34, 3625 (1986).

${ }^{20}$ J. B. Marion, Classical Dynamics of Particles and Systems, 2nd ed. (Academic Press, New York and London, 1970).

21 M. Christandl, N. Datta, A. Ekert, and A. J. Landahl, Phys. Rev. Lett. 92, 187902 (2004).

22 P. Jordan and E. Wigner, Z. Physik 47, 631 (1928).

${ }^{23}$ G. Floquet, Annales scientifiques de l'École Normale Supérieure 12, 47 (1883)

${ }^{24}$ P. Hänggi, in Quantum Transport and Dissipation, edited by T. Dittrich, P. Hänggi, G.-L. Ingold, B. Kramer, G. Schön, and W. Zwerger (Wiley-VCH, Weinheim, 1998) Chap. 5, pp. 249-286.

25 P. Kuchment, Floquet Theory for Partial Differential Equations (Birkhäuser Verlag, 1993).

${ }^{20}$ J. Daleckii and M. Krein, Stability of Solutions of Differential Equations in Banach Space, Translations of mathematical monographs (American Mathematical Society, 2002).

27 I. Panardo, Stability of periodic systems and Floquet The- ory, Master's thesis, Università degli Studi di Padova (2014).

28 A. R. Kolovsky and H. J. Korsch, J. Sib. Fed. Univ. Math. Phys. 3, 311 (2010).

29 D. H. Dunlap and V. M. Kenkre, Phys. Lett. A 127, 438 (1988).

30 D. J. Luitz, A. Lazarides, and Y. Bar Lev, PRB 97, 020203(R) (2018).

31 M. Grifoni and P. Hänggi, Phys. Rep. 304, 229 (1998).

32 E. Lieb, T. Schultz, and D. Mattis, Ann. Phys. 16, 407 (1961).

33 S. Katsura, Phys. Rev. 127, 1508 (1962).

34 A. Kay, Int. J. Quant. Inf 8, 641 (2010).

35 M. Christandl, N. Datta, T. C. Dorlas, A. Ekert, A. Kay, and A. J. Landahl, Phys. Rev. A 71, 032312 (2005)

36 J. Van der Jeugt, Journal of Physics: Conference Series 284, 012059 (2011)

R. Chakrabarti and J. Van der Jeugt, Journal of Physics A: Mathematical and Theoretical 43, 085302 (2010)

38 R. Koekoek and R. F. Swarttouw, The Askey-scheme of hypergeometric orthogonal polynomials and its q-analogue, Tech. Rep. 98-17 (Delft University of Technology, 1998).

39 A. Erdélyi, W. Magnus, F. Oberhettinger, and F. Tricomi, Higher transcendental functions, Vol. 1 (New York, NY, McGraw-Hill, 1953) pp. 82-85.

40 G. M. Nikolopoulos, D. Petrosyan, and P. Lambropoulos, Journal of Physics: Condensed Matter 16, 4991 (2004).

41 C. Albanese, M. Christandl, N. Datta, and A. Ekert, Phys. Rev. Lett. 93, 230502 (2004)

${ }^{42}$ P. Karbach and J. Stolze, Phys. Rev. A 72, 030301(R) (2005).

43 T. Shi, Y. Li, Z. Song, and C.-P. Sun, Phys. Rev. A 71, $032309(2005)$

${ }^{44}$ X. Q. Xi, J. B. Gong, T. Zhang, R. H. Yue, and W. M. Liu, The European Physical Journal D 50, 193 (2008).

45 M. Bruderer, K. Franke, S. Ragg, W. Belzig, and D. Obreschkow, Phys. Rev. A 85, 022312 (2012).

46 J. Stolze, G. A. Álvarez, O. Osenda, and A. Zwick, in Quantum State Transfer and Quantum Network Engineering, Quantum Science and Technology, edited by G. M. Nikolopoulos and I. Jex (Springer, Berlin, 2014).

47 P. Lorenz and J. Stolze, Phys. Rev. A 90, 044301 (2014).

48 C. Creffield, Phys. Rev. B 67, 165301 (2003).

49 C. Creffield, EPL 66, 631 (2004).

50 J. v. Neumann and E. Wigner, Phys. Zeits. 30, 467 (1929).

51 The shape of the peaks in $\left\langle f_{0 N}(t)\right\rangle$ is plausible by analogy to the system without external drive. There, the energy eigenfunction amplitudes along the chain possess an approximately Gaussian envelope and the energy spectrum is strictly equidistant. From that, $\left|f_{0 N}(t)\right|^{2}$ can be shown to be approximately given by the Jacobian theta function $\vartheta_{4}$ (which is periodic), whose individual peaks have nearperfect Gaussian shape and width proportional to $N^{-\frac{1}{2}}$. 